Özgen, I.; Zhao, J.; Liang, D.; Hinkelmann, R.

\title{
Urban flood modeling using shallow water equations with depth-dependent anisotropic porosity
}

Journal article | Accepted manuscript (Postprint)

This version is available at http://dx.doi.org/10.14279/depositonce-7232

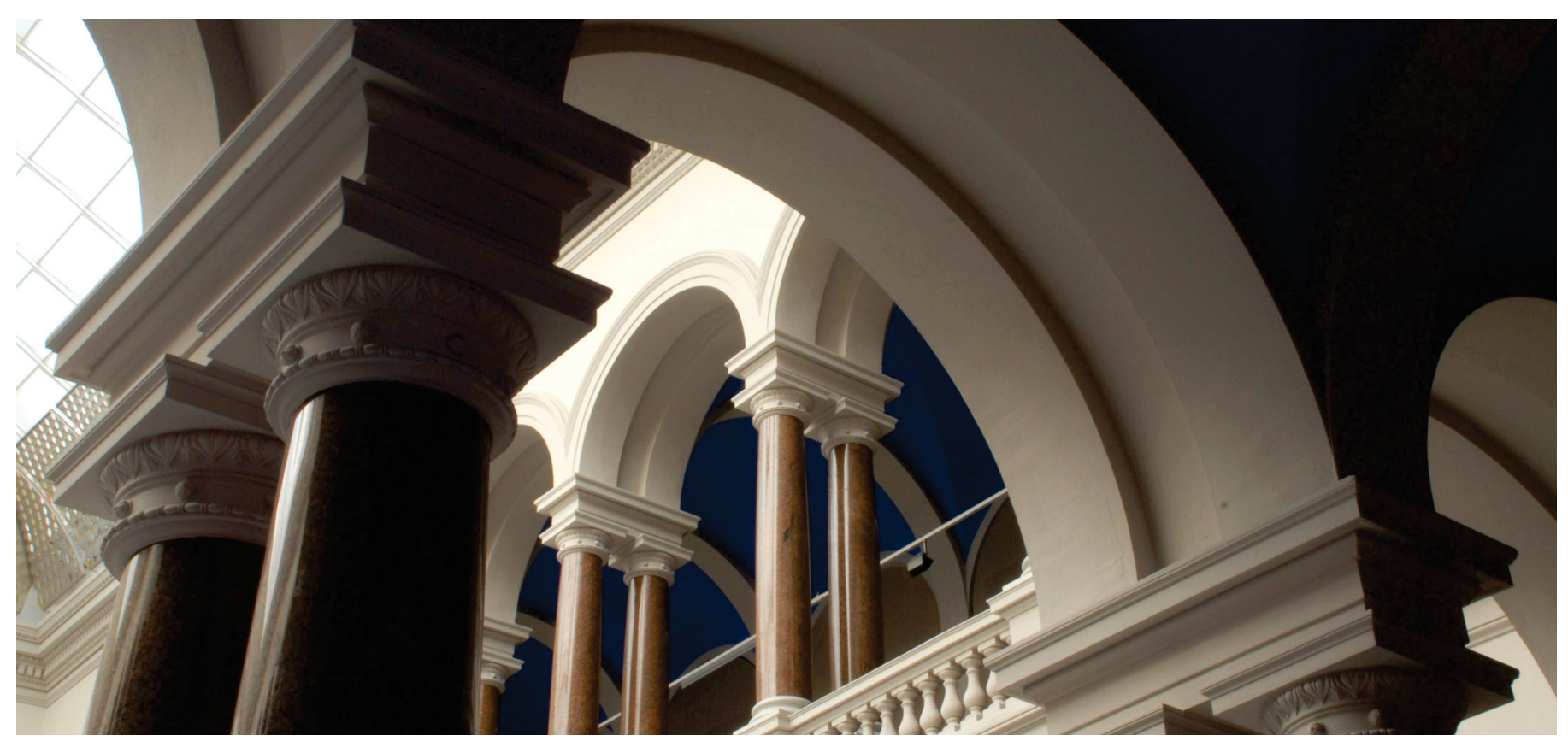

Özgen, I., Zhao, J., Liang, D., \& Hinkelmann, R. (2016). Urban flood modeling using shallow water equations with depth-dependent anisotropic porosity. Journal of Hydrology, 541, 1165-1184. https://doi.org/10.1016/j.jhydrol.2016.08.025 


\title{
Urban flood modeling using shallow water equations with depth-dependent anisotropic porosity
}

\author{
Ilhan Özgen $^{\mathrm{a}, *}$, Jiaheng Zhao ${ }^{\mathrm{a}}$, Dongfang Liang ${ }^{\mathrm{b}}$, Reinhard Hinkelmann ${ }^{\mathrm{a}}$ \\ ${ }^{a}$ Chair of Water Resources Management and Modeling of Hydrosystems, Technische Universität Berlin, Germany \\ ${ }^{\mathrm{b}}$ Department of Engineering, University of Cambridge, UK
}

\begin{abstract}
A B S T R A C T
The shallow water model with anisotropic porosity conceptually takes into account the unresolved subgrid-scale features, e.g. microtopography or buildings. This enables computationally efficient simulations that can be run on coarser grids, whereas reasonable accuracy is maintained via the introduction of porosity. This article presents a novel numerical model for the depth-averaged equations with anisotropic porosity. The porosity is calculated using the probability mass function of the subgrid-scale features in each cell and updated in each time step. The model is tested in a one-dimensional theoretical benchmark before being evaluated against measurements and high-resolution predictions in three case studies: a dam-break over a triangular bottom sill, a dam-break through an idealized city and a rainfall-runoff event in an idealized urban catchment. The physical processes could be approximated relatively well with the anisotropic porosity shallow water model. The computational resolution influences the porosities calculated at the cell edges and therefore has a large influence on the quality of the solution. The computational time decreased significantly, on average three orders of magnitude, in comparison to the classical high-resolution shallow water model simulation.
\end{abstract}

\section{Introduction}

In shallow water modeling of river hydraulics (Özgen et al., 2013; Kesserwani and Liang, 2015), urban flooding (Liang, 2010; Mignot et al., 2006), urban runoff (Cea et al., 2010; Liang et al., 2007; Liang et al., 2015) and rainfall-runoff on natural environments (Mügler et al., 2011; Özgen et al., 2015; Simons et al., 2014; Viero et al. 2014), the topographical features have a large influence on the numerical results. The availability of digital elevation data has increased significantly due to recent improvements in surveying technology, notably laser scanning and light detection and ranging (LIDAR) technologies, which provide high-resolution data sets at relatively low cost (Gessner et al., 2014; Gourbesville, 2009). However, mainly due to computational constraints, incorporating these data sets into shallow water models is challenging (McMillan and Brasington, 2007; Dottori et al., 2013). The difficulty arises from multiple scales in the physical processes. For example, in a small natural catchment with a scale of around a square kilometer, local depressions and microtopogra-

\footnotetext{
* Corresponding author at: Secr. TIB1-B14, Chair of Water Resources Management and Modeling of Hydrosystems, Technische Universität Berlin, Gustav-MeyerAllee 25, 13353 Berlin, Germany.

E-mail address: ilhan.oezgen@wahyd.tu-berlin.de (I. Özgen).
}

phy with horizontal scales less than a square meter influence the flow field significantly (Blöschl and Sivapalan, 1995; Dunne et al., 1991; Thompson et al.). Similarly, in urban flood models the city may spread up to several hundred square kilometers but the flood flow can be diverted, slowed down or completely blocked by manmade structures, e.g. buildings, bridges or walls, whose characteristic scale are in meters. In order to accurately capture the effect of microtopography or buildings, they have to be included in the discretization. Due to the co-existence of multiple scales, this leads to extremely large computational mesh, which requires large data storage, large number of operations per time step, small time step size and thus large computational effort. In fact, the computational cost is inversely proportional to the third power of the cell size (Kim et al., 2014). Therefore, practical applications have to compromise between spatial accuracy and computational efficiency (Lacasta et al., 2014) and are often carried out on supercomputers (Smith and Liang, 2013).

For super-computers, high-performance parallel computation methods on shared or distributed memory have been developed in literature (Hinkelmann, 2005) and very recently graphic processing units have been exploited for scientific computation (e.g., Lacasta et al., 2014; Lacasta et al., 2015; Smith and Liang, 2013).

A different approach to speed up simulations is to conceptually account for small scale ground variations without explicitly dis- 
cretizing them (McMillan and Brasington, 2007). This allows to run the simulations on coarser meshes. In this context, the shallow water equations with porosity have been initially developed by Defina et al. (1994) and Defina (2000) to account for microtopography in partially inundated cells. Here, a single porosity is assigned to each cell, which represents the fraction of the cell that contributes to the flow. The porosity is calculated by a distribution function, which returns the porosity depending on the water depth in the cell. The distribution function is defined for the whole domain. In Viero et al. 2014, Defina's porous shallow water equations are applied to coupled simulations of surface and subsurface flows in natural catchments.

The porosity concept was also applied to urban flood modeling by Hervouet et al. (2000) to account for buildings. Significant contribution to the porosity concept in the context of urban flood modeling was made by Guinot and Soares-Frazão (2006), Soares-Frazão et al. (2008) and Guinot (2012). Because the buildings in urban flood models are usually not fully submerged during the flood event, the area available for the flow stays constant during the simulation. Consequently, most porous urban flood models assign a constant porosity to each cell which only depends on the fraction of the cell occupied by buildings. An exception is the urban flood model presented in Henonin et al. (2015), wherein the authors calculate the inundated area of each cell according to the water elevation and use it in the mass balance. Although the authors do not explicitly use porosity terms, the model in Henonin et al. (2015) is essentially equivalent to a single porosity model with a depth-dependent porosity. The same strategy for porosity calculation is followed in this work. Further studies regarding the shallow water equations with single porosity in the context of urban flooding were carried out in Cea and Vázquez-Cendón (2009), Garrido et al. (2011), Mohamed (2014), Soares-Frazão et al. (2008) and Velickovic et al. (2010). Single porosity shallow water models can not differentiate between spatial directions. The flow in all directions is governed by the same porosity. However, buildings in urban flood models usually have a directionality which leads to preferential flow paths of the water. Therefore, Sanders et al. (2008) introduced the anisotropic porosity shallow water model, wherein a volumetric porosity inside the cell is defined to account for the fraction of the cell available for water. In addition an areal porosity is assigned to each cell edge which describes the conveyance there (Sanders' model). The equations were derived using the integral form of the shallow water equations, thus these equations can be solved only by a finite volume method. Sanders' model was further investigated in Kim et al. $(2015,2014)$ and Schubert and Sanders (2012). In Özgen et al. (2016) a modified version of Sanders' model that allows full submergence of unresolved topographic features by introducing a mutual dependency between water depth and porosity is derived.

This article presents a numerical model to solve the equations derived in Özgen et al. (2016) on Cartesian grids. The main difference from Sanders' model is that submergence of unresolved topography leads to a different formulation of the porosities depending on the water depth in the cell. The main contribution of this work is the discussion on discretizing the porosity terms in the cell and at the edge and the illustration of the model's behavior via detailed case studies. In the present model, each cell and each edge are automatically assigned an individual porosity that depends on the water depth and the underlying topography. Thus, the model is automatically adjusted based on the computational mesh. The model performance is investigated in a theoretical test case. Then, case studies of laboratory experiments are presented to further investigate the model's behavior.

\section{Governing equations}

The two-dimensional shallow water equations with anisotropic porosity can be written in integral-differential form as:

$\frac{\partial}{\partial t} \int_{\Omega} i \mathbf{q} d \Omega+\oint_{\partial \Omega} i \mathbf{F n} d r=\int_{\Omega} i \mathbf{s} d \Omega+\oint_{\partial \Omega^{*}} \mathbf{s}^{*} d r^{*}$

Here, $\Omega$ is the total base area of the control volume, $\partial \Omega$ is the boundary of the control volume, $r$ is the path along the boundary $\partial \Omega, \partial \Omega^{*}$ is the boundary between the fluid and the solid inside the control volume and $r^{*}$ is the path along this boundary (cf. Sanders et al., 2008; Özgen et al., 2016). $i$ is the so-called phase function, defined as:

$i(x, y)= \begin{cases}1, & \eta(x, y)>z_{b}(x, y) \\ 0, & \text { else }\end{cases}$

$\eta$ is the water elevation, $z_{b}$ is the bottom elevation, $\mathbf{q}$ is the vector of conserved variables, $\mathbf{s}$ is the source term vector, $\mathbf{F}$ is the flux vector and $\mathbf{n}=\left[n_{x}, n_{y}\right]^{T}$ is the normal vector of the boundary, with $n_{x}$ and $n_{y}$ are the components of the normal vector in $x$-and $y$-directions of the Cartesian coordinate system, respectively. Fig. 1 illustrates the phase function, $\eta$ and $z_{b}$. The vectors $\mathbf{q}$ and $\mathbf{s}$ are expressed as:

$\mathbf{q}=\left[\begin{array}{c}h \\ q_{x} \\ q_{y}\end{array}\right], \quad \mathbf{s}=\left[\begin{array}{c}i_{r} \\ s_{b, x}+s_{f, x} \\ s_{b, y}+s_{f, y}\end{array}\right]$

Here, $h=\eta-z_{b}$ stands for water depth, $q_{x}$ and $q_{y}$ are the unit discharges in $x$ - and $y$-directions, respectively. $i_{r}$ is the mass source term, e.g. rainfall intensity; $s_{b, x}, s_{b, y}$ are the bed slope source terms

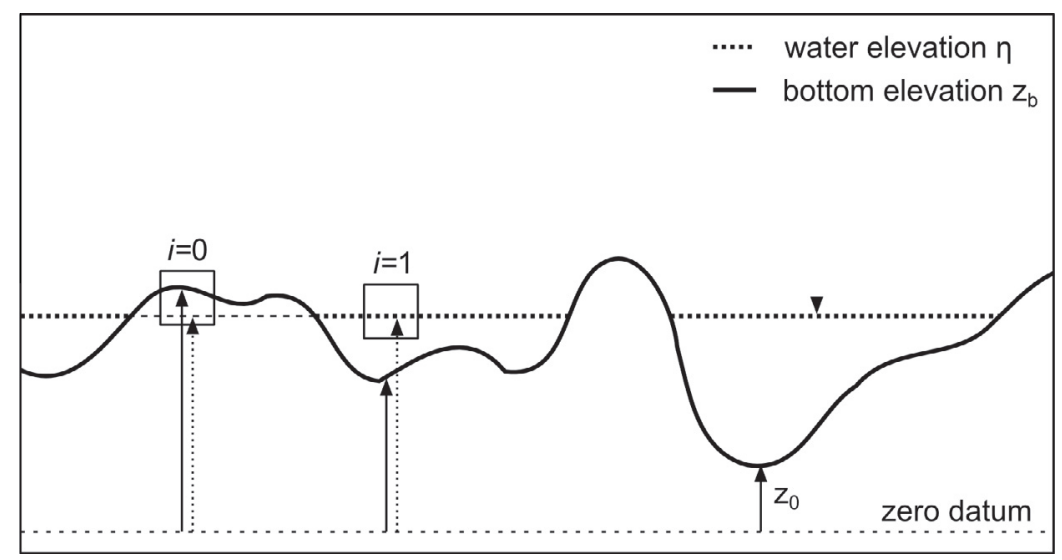

Fig. 1. Definition of phase function $i$, water elevation $\eta$ (dashed), bottom elevation $z_{b}$ (black) and zero datum $z_{0}$ in a vertical section through a control volume. 
in $x$ - and $y$-directions, respectively which account for variations in bottom, $s_{f, x}, s_{f, y}$ are the friction source terms in $x$ - and $y$-directions, respectively:

$s_{b, x}=-g h \frac{\partial z_{b}}{\partial x}, \quad s_{b, y}=-g h \frac{\partial z_{b}}{\partial y}$,

$s_{f, x}=-c_{f} q_{x} \frac{\sqrt{q_{x}^{2}+q_{y}^{2}}}{h^{2}}, \quad s_{f, y}=-c_{f} q_{y} \frac{\sqrt{q_{x}^{2}+q_{y}^{2}}}{h^{2}}$

$c_{f}$ is the Chézy roughness coefficient, which can be expressed via Manning's law:

$c_{f}=g n^{2} h^{-1 / 3}$

$n$ is Manning's roughness coefficient and $g$ is the gravitational acceleration. The flux vector is often split into its $x$ - and $y$-component:

$\mathbf{F n}=\mathbf{f} n_{x}+\mathbf{g} n_{y}$

f and $\mathbf{g}$ are defined as:

$\mathbf{f}=\left[\begin{array}{c}q_{x} \\ u q_{x}+0.5 g h^{2} \\ u q_{y}\end{array}\right], \quad \mathbf{g}=\left[\begin{array}{c}q_{y} \\ v q_{x} \\ v q_{y}+0.5 g h^{2}\end{array}\right]$

Here, $u$ and $v$ are the velocities in $x$ - and $y$-directions, respectively. Finally, $\mathbf{s}^{*}$ is the source vector accounting for fluid pressure along the interface $\partial \Omega^{*}$. The calculation of $\mathbf{s}^{*}$ is non-trivial and will be addressed in the next section.

\section{Numerical model}

\subsection{Finite volume formulation of the equations}

The integral-differential form of the shallow water equations can be solved with the finite volume method. However, the phase function $i$ can not be evaluated explicitly in the finite volume cell, because the bottom elevation inside the cell is not resolved. Therefore, the integral terms on the left hand side of Eq. (1) have to be calculated with the concept of porosity.

In Özgen et al. (2016), the volumetric porosity is defined as:

$\phi=\frac{\int_{\Omega} i\left(\eta-z_{b}\right) d \Omega}{\int_{\Omega}\left(\eta-z_{0}\right) d \Omega}$

The areal porosity is calculated as:

$\psi=\frac{\oint_{\partial \Omega} i\left(\eta-z_{b}\right) d r}{\oint_{\partial \Omega}\left(\eta-z_{0}\right) d r}$

Here, $z_{0}$ is the elevation of the lowest point inside the control volume with regard to a datum. Both are illustrated in Fig. 1. Evaluating the integral terms leads to modified flux and storage vectors (Özgen et al., 2016). Rewriting the line integral as a sum over the finite volume edges transforms Eq. (1) to:

$\frac{\partial}{\partial t}(\phi \Omega \overline{\mathbf{q}})+\sum_{k} \psi_{k} r_{k} \widehat{\mathbf{F}}_{k} \mathbf{n}_{k}=\int_{\Omega} i \mathbf{s} d \Omega+\oint_{\partial \Omega^{*}} \mathbf{s}^{*} d r$

$k$ is the index of the path integral and $r_{k}$ is the length of the integration path. The storage vector $\mathbf{q}$ in Equation 3 is rewritten as:

$\overline{\mathbf{q}}=\left[\begin{array}{c}\left(\bar{\eta}-z_{0}\right) \\ \bar{u}\left(\bar{\eta}-z_{0}\right) \\ \bar{v}\left(\bar{\eta}-z_{0}\right)\end{array}\right]$

The bar over a variable indicates volume-averaged variables which are constant within the cell:
$\bar{\eta}=\frac{\int_{\Omega} i \eta d \Omega}{\int_{\Omega} i d \Omega}, \quad \overline{\mathbf{v}}=\frac{\int_{\Omega} i h \mathbf{v} d \Omega}{\int_{\Omega} i h d \Omega}$

If $i=0$ over the whole control volume, the averaging is not carried out and the volume-averaged variables are taken to be $\bar{\eta}=0$ and $\overline{\mathrm{v}}=0$. The flux vector in Eq. (3) is rewritten as:

$\widehat{\mathbf{F}} \mathbf{n}=\left[\begin{array}{c}\hat{u}\left(\hat{\eta}-z_{0}\right) n_{x}+\hat{v}\left(\hat{\eta}-z_{0}\right) n_{y} \\ \hat{u} \hat{u}\left(\hat{\eta}-z_{0}\right) n_{x}+0.5 g\left(\hat{\eta}-z_{0}\right)^{2} n_{x}+\hat{u} \hat{v}\left(\hat{\eta}-z_{0}\right) n_{y} \\ \hat{v} \hat{u}\left(\hat{\eta}-z_{0}\right) n_{x}+\hat{v} \hat{v}\left(\hat{\eta}-z_{0}\right) n_{y}+0.5 g\left(\hat{\eta}-z_{0}\right)^{2} n_{y}\end{array}\right]$

The circumflex over a variable indicates area-averaged variables at the edge:

$\hat{h}=\frac{\int_{r} i h d r}{\int_{r} i d r}, \quad \hat{\eta}=\frac{\int_{r} i \eta d r}{\int_{r} i d r}, \quad \hat{\mathbf{v}}=\frac{\int_{r} i h \mathbf{v} d r}{\int_{r} i h d r}$

As before, if $i=0$ over the whole edge the averaging is not carried out and all variables are taken to be nil. Then, Eq. (11) can be solved with a suitable time integration method.

\subsection{Porosity computation}

In order to calculate the porosities, the Probability Mass Function (PMF) of the unresolved bottom elevation inside the cell is calculated in the pre-processing step. The PMF is defined as the probability density function with discrete variables and can be computed by sampling the bottom elevation at a resolution much higher than the computational mesh. This assumes that the bottom elevation data is resolved at the finer resolution than the computational mesh resolution. The PMF is calculated for each cell and each edge seperately. In the context of this work, the PMF value of a certain elevation corresponds to the fraction of area below this elevation over the total area of the cell or the fraction of length of the edge below the specified elevation over the total length. Then, for any given water elevation $\bar{\eta}$, the volumetric porosity $\phi$ can be calculated as:

$\phi(\bar{\eta})=\frac{1}{\bar{\eta} \Omega} \sum_{i}^{N} \min \left(0, \bar{\eta}-z_{b, i}\right) \operatorname{PMF}\left(z_{b, i}\right) \Omega_{i}$

Here, $i$ is the index of bottom elevation $z_{b, i} \operatorname{PMF}\left(z_{b, i}\right)$ is the value of the PMF evaluated at $z_{b, i}$. In the present numerical model, the class index increases as the bottom elevation increases, i.e. the lowest bottom elevation corresponds to the smallest class index and the highest bottom elevation corresponds to the largest class index. $N$ denotes the total number of classes. Similarly, the areal porosity $\psi$ at one edge is computed as:

$\psi(\hat{\eta})=\frac{1}{\hat{\eta} \Delta k} \sum_{i}^{N} \min \left(0, \hat{\eta}-z_{b, i}\right) \operatorname{PMF}\left(z_{b, i}\right) \Delta k_{i}$

$\Delta k$ is the length of the edge. The PMF for the edge is sampled from the subgrid cells adjacent to the edge under consideration. Because the adjacent neighbor cell also contributes to the porosity of the edge. The samples at the edges are modified as:

$$
\begin{cases}z_{b, i}^{L}=z_{b, i}^{R}, & \text { if } z_{b, i}^{L}<z_{b, i}^{R} \\ z_{b, i}^{R}=z_{b, i}^{L}, & \text { if } z_{b, i}^{L}>z_{b, i}^{R}\end{cases}
$$

Here, the superscripts $L$ and $R$ denote the left and right sides of the edge, respectively. The idea is to take clustering effects and cell blockage which have been reported in Yu and Lane (2006) and Yu and Lane (2006) into account. The PMF is computed for each cell and edge once in the pre-processing step and is stored. Once the PMF is obtained, the mesh used for sampling is discarded and therefore the information of the high-resolution bottom elevation is not available anymore. The bottom elevation of each computational cell 
is set at the lowest value found from the high-resolution mesh. Additionally, the elevation at each edge is stored and used in the subsequent computation. The porosities are updated at the beginning of each time step according to Eqs. (16) and (17). It is noted that in Equation 16 and 17 each sample is weighted equally. This assumes that each sample represents an equal amount of area. This is easy to assume for either square-shaped or rectangular-shaped grid cells if the subgrid-scale elevations are evenly distributed. For a triangular cell, evenly distributed subgrid-scale bottom elevations would not represent equal areas and the equations must be further modified to account for this. One approach would be to perform a Voronoi-tessellation in each cell to calculate weights for each sample. In this study, only structured grids with squareshaped cells are used.

\subsubsection{Choice of water elevation for areal porosity calculation}

The areal porosity at the edge is calculated according to the water elevation at the edge. Because the edge is an interface between two neighboring cells, a choice between two water elevations has to be made to calculate the areal porosity, namely the water elevation at the left $\hat{\eta}_{L}$ and the water elevation at the right $\hat{\eta}_{R}$ of the edge. In this work, the upstream water elevation is chosen for porosity calculation. For example, if the case illustrated in Fig. 2 is considered, the areal porosity $\psi$ will be computed according to the water elevation on the left side of the edge $\hat{\eta}_{L}$. In Fig. $2, \hat{z}_{b}$ is the bottom elevation at the edge. The calculation of $\hat{z}_{b}$ is discussed in the next section (Section 3.3).

\subsection{Flux computation}

The numerical scheme is a Godunov-type explicit finite volume scheme with second order MUSCL reconstruction (van Leer, 1979). Values at cell center are linearly extrapolated to the edges, whereby the slope of the extrapolation function is limited by a min-mod slope limiter (Hou et al., 2012). The reconstructed values are used to calculate the numerical fluxes over the cell edge by solving the Riemann problem at the edge using a Harten, Lax and van Leer approximate Riemann solver with the contact wave restored (HLLC) (Toro et al., 1994). As suggested in Audusse et al. (2004), only $\hat{\eta}, \hat{\mathbf{q}}$ and $\hat{h}$ are extrapolated. At wet-dry interfaces, the MUSCL reconstruction is omitted to ensure numerical stability (Liang, 2010; Hou et al., 2013; Liang and Borthwick, 2009).

The reconstruction of the bottom elevation at the edge differs slightly from most reconstructions (e.g., Audusse et al., 2004; Hou et al., 2013). In a first step, the bottom elevation at the edge $z_{b, i}^{\text {rec }}$ is calculated as

$z_{b, i}^{r e c}=\hat{\eta}_{i}-\hat{h}_{i}$

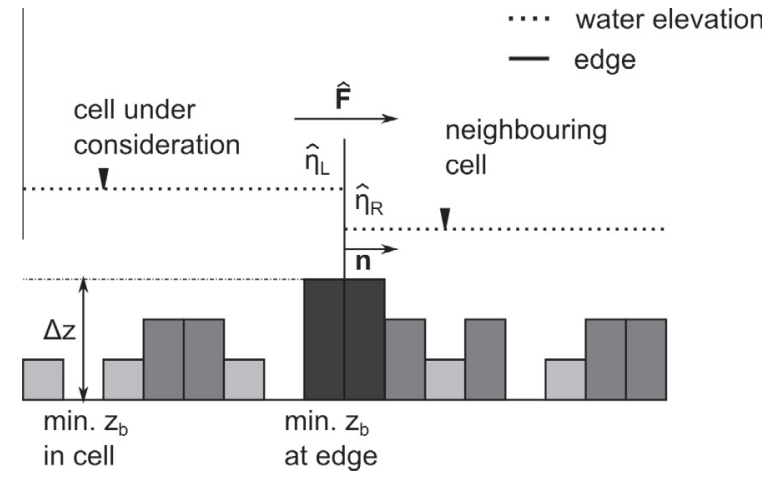

Fig. 2. Side view of two neighboring cells for the choice of the water elevation to calculate $\psi$, the cell under consideration is on the left side, water elevation is dashed line, definitions of $\Delta z, \mathbf{n}, \hat{\eta}_{L}$ and $\hat{\eta}_{R}$.
In an additional second step the difference between the lowest bed elevation at the edge and the bottom elevation of the cell is calculated:

$\Delta z_{i}=z_{b}^{\text {edge }}-z_{b, i}^{\text {cell }}$

$z_{b}^{\text {edge }}$ refers to the lowest elevation at the edge and $z_{b, i}^{\text {cell }}$ refers to the bottom elevation of the cell on the left or right side of the edge (cf. Fig. 2). Then, $\Delta z_{i}$ is added to $z_{b, i}^{r e c}$ :

$\hat{z}_{b, i}=z_{b, i}^{r e c}+\Delta z_{i}$

The reconstruction carried out for the left and right side of the edge gives $\hat{\eta}_{L}, \hat{\mathbf{q}}_{L}, \hat{h}_{L}, \hat{z}_{b, L}, \hat{\eta}_{R}, \hat{\mathbf{q}}_{R}, \hat{h}_{R}, \hat{z}_{b, R}$. Hereinafter, the cell on the left side of the edge is assumed to be the cell under consideration. Then, the non-negative water depth reconstruction (Audusse et al., 2004) is carried out as follows: The bottom elevation at the edge is defined as:

$\hat{z}_{b}=\max \left(\hat{z}_{b, L}, \hat{z}_{b, R}\right)$

Water elevation on the left side of the edge and the bottom elevation at the edge are compared and the lower value is set as the new bottom elevation.

$\hat{z}_{b}=\min \left(\hat{z}_{b}, \hat{\eta}_{L}\right)$

Water depths are reconstructed as:

$\hat{h}_{R}=\max \left(0, \hat{\eta}_{R}-\hat{z}_{b}\right)-\max \left(0, \hat{z}_{b, R}-\hat{z}_{b}\right), \quad \hat{h}_{L}=\hat{\eta}_{L}-\hat{z}_{b}$

The vector of velocities at the left and right sides of the edge $\left(\hat{\mathbf{v}}_{i}=\left[\hat{u}_{i}, \hat{v}_{i}\right]^{T}\right)$ are calculated as:

$\hat{\mathbf{v}}_{i}= \begin{cases}0, & \hat{h}_{i}<\epsilon \\ \hat{\mathbf{q}}_{i} / \hat{h}_{i}, & \hat{h}_{i} \geqslant \epsilon\end{cases}$

$\epsilon$ is a threshold to avoid division by 0 and further indicates whether a cell is considered wet or dry. In this work it is chosen $\epsilon=10^{-6} \mathrm{~m}$. Finally, $\hat{h}_{L}, \hat{\mathbf{v}}_{L}, \hat{h}_{R}$ and $\hat{\mathbf{v}}_{R}$ are used by the HLLC Riemann solver to compute the flux over the edge.

\subsection{Source term computation}

\subsubsection{Bed slope and friction source term computation}

In Eq. (1), three source terms have to be numerically solved: the bed slope source term, the friction source term and the solid-fluid interfacial pressure source term. The first two source terms occur as a result of depth-averaging and can be found also in the classical two-dimensional shallow water equations. The last term results from the ground unevenness not resolved by the computational mesh and is discussed in Sanders et al. (2008) and Özgen et al. (2016).

The bed slope source term can be written as

$\mathbf{s}_{b}=\left[\begin{array}{c}0 \\ s_{b, x} \\ s_{b, y}\end{array}\right]$

where the definitions of the terms are given in Eq. (4). In Valiani and Begnudelli (2006), the divergence form for bed slope is presented, which transforms the bed slope source term within the cell into a flux term over its edges:

$\int_{\Omega} i \mathbf{s}_{b} d \Omega=\oint_{\partial \Omega} i \mathbf{F}_{b} \mathbf{n} d r$

The integral is evaluated and the line integral is approximated by the algebraic expression: 
$\oint_{\partial \Omega} i \mathbf{F}_{b} \mathbf{n} d r=\sum_{k} \psi_{k} r_{k} \widehat{\mathbf{F}}_{b} \mathbf{n}_{k}$

Hou et al. (2013) propose an extension of this approach to higher order accuracy by dividing the integral over the cell into integrals over subcells. This allows non-linear variations of bed elevation, which is suitable for the model presented in this work because separate bottom elevations are defined at the cell edges. The vector of bed slope flux at edge $k$ is written as:

$$
\mathbf{F}_{b, k} \mathbf{n}_{k}=\left[\begin{array}{c}
0 \\
-0.5 n_{x} g\left(h_{k}+\bar{h}\right)\left(\hat{z}_{b, k}-\bar{z}_{b}\right) \\
-0.5 n_{y} g\left(h_{k}+\bar{h}\right)\left(\hat{z}_{b, k}-\bar{z}_{b}\right)
\end{array}\right]
$$

Using Eq. (10), the evaluation of the integral in Eq. (28) over edge $k$ in $x$-direction gives:

$$
\begin{aligned}
& \int_{\partial \Omega_{k}}-0.5 \sin _{x} g\left(h_{k}+\bar{h}\right)\left(\hat{z}_{b, k}-\bar{z}_{b}\right) d r \\
& \quad=-0.5 g\left(\hat{z}_{b, k}-\bar{z}_{b}\right) \int_{\partial \Omega_{k}} g\left(i h_{k}+i \bar{h}\right) d r \\
& \left.\quad=-0.5 g\left(\hat{z}_{b, k}-\bar{z}_{b}\right) \quad \psi_{k}\left(\hat{\eta}_{k}-z_{0}\right) r_{k}+\int_{\partial \Omega_{k}} i \bar{h} d r\right)
\end{aligned}
$$

The latter integral in Eq. (30) is approximated with:

$\int_{\partial \Omega_{k}} i \bar{h} d r \approx \psi_{k} \bar{h} r_{k}$

The evaluation of the integral in $y$-direction is similar. Then, the evaluated bottom slope flux vector $\widehat{\mathbf{F}}_{b, k} \mathbf{n}_{k}$ over the edge $k$ can be written as:

$$
\widehat{\mathbf{F}}_{b, k} \mathbf{n}_{k}=\left[\begin{array}{c}
0 \\
-0.5 n_{x} g\left(\hat{\eta}_{k}-z_{0}+\bar{h}\right)\left(\hat{z}_{b, k}-\bar{z}_{b}\right) \\
-0.5 n_{y} g\left(\hat{\eta}_{k}-z_{0}+\bar{h}\right)\left(\hat{z}_{b, k}-\bar{z}_{b}\right)
\end{array}\right]
$$

For the friction source term, the standard expression of the friction source vector as introduced in Eq. (5) is used. The term is discretized in a point implicit way as shown in Simons et al. (2014).

\subsubsection{Solid-fluid interfacial pressure source term computation}

The solid-fluid interfacial pressure source term treatment follows the modeling concept in Sanders et al. (2008). The term is split into a stationary and non-stationary part:

$\oint_{\partial \Omega^{*}} \mathbf{s}^{*} d r=\oint_{\partial \Omega^{*}} \mathbf{s}_{s t}^{*} d r+\int_{\Omega} i \mathbf{s}_{n s}^{*} d \Omega$

The stationary part balances the pressure and flux terms as the flow converges to a stationary state and the non-stationary part results from the water elevation fluctuation inside the computational cell that can not be resolved (Sanders et al., 2008). The non-stationary term $\mathbf{s}_{n s}^{*}$ is integrated over the cell. In Sanders et al. (2008) and Özgen et al. (2016), this term follows a generalized drag law proposed in Nepf (1999):

$\mathbf{s}_{n s}^{*}=\left[\begin{array}{c}0 \\ c_{D} \bar{u} \sqrt{\bar{u}^{2}+\bar{v}^{2}} \\ c_{D} \bar{u} \sqrt{\bar{u}^{2}+\bar{v}^{2}}\end{array}\right]$

$c_{D}$ is the dimensionless drag coefficient, which is calculated with:

$c_{D}=0.5 c_{D}^{0} a \cdot \min \left(h, z_{b}^{\max }-z_{b}^{\min }\right)$

The parameter $a$ represents the projected width of the obstruction facing the flow per unit planform area and depends on the angle of attack and width of the obstacle (Sanders et al., 2008). $c_{D}^{0}$ is a reference drag coefficient obtained by calibration, and $a$ is a modifica- tion coefficient. In theory, it is possible to determine $a$ exactly from the geometry data and calibrate only $c_{D}^{0}$, yet this is not done in this work. Instead, the model is calibrated using the product $c_{D}^{0} \cdot a$. The reason for this is that calculating the angle of attack for the value of $a$ during the simulation is not trivial. In addition, the value of $c_{D}^{0}$ depends on the Reynolds number and the shape of the obstacle. In Nepf (1999) and Sanders et al. (2008), it is suggested that the value of $a$ should be estimated in a predictor step and then updated in a corrector step based on the flow values of the predictor step. This approach is not followed in this work, because it requires extra knowledge of the subgrid-scale obstacles beyond the porosity function, i.e. information about the shape and the directionality of the obstacles have to be stored. An additional challenge is that the values of $a$ and $c_{D}^{0}$ depend on the water depth in the cell, as the geometry of the obstacles might vary in the vertical direction. The full assessment of the present approach requires additional research. Additionally, the value $c_{D}^{0} \cdot a$ is assumed constant over the whole domain, because the cases investigated are relatively simple. However, each cell could also be assigned a separate $c_{D}^{0} \cdot a$. This would allow a better representation of the heterogeneity in the domain, but the drawback is that the model calibration becomes very complicated and requires large quantities of data. This further suggests that a more precise definition of both $a$ and $c_{D}^{0}$ is required. Overall, the calculation of the non-stationary term needs further research.

The stationary part of the interfacial pressure source term is essential, as it well-balances the scheme. Here, the vector of the stationary interfacial pressure source term is derived by evaluating the C-property of the scheme. This leads to the same formulation as in Sanders et al. (2008):

$\oint_{\partial \Omega^{*}} \mathbf{s}_{s t}^{*} d r=\sum_{k} \psi_{k} \widehat{\mathbf{F}}_{*, k} \mathbf{n}_{k} r_{k}$

with:

$\widehat{\mathbf{F}}_{*, k}=\left[\begin{array}{c}0 \\ 0.5 \bar{h}^{2} n_{k, x} \\ 0.5 \bar{h}^{2} n_{k, y}\end{array}\right]$

The proof of C-property is trivial and omitted for sake of brevity.

\subsection{Time integration}

A two-stage total variation diminishing Runge-Kutta method (Gottlieb and Shu, 1996) is used. The values at next time step $n+1$ are calculated in two stages. The first stage is

$\tilde{\phi}^{n+1} \tilde{\mathbf{q}}^{n+1}=\phi^{n} \mathbf{q}^{n}-\Delta t \sum_{k} \psi_{k}^{n} \widehat{\mathbf{F}}_{t o t, k}^{n} r_{k} \mathbf{n}_{k}+\Delta t \phi^{n}\left(\mathbf{s}^{n}+\mathbf{s}_{n s}^{*, n}\right) \Omega$

and the final value is then calculated as

$\left.\phi^{n+1} \mathbf{q}^{n+1}=0.5 \quad \phi^{n} \mathbf{q}^{n}+\tilde{\phi}^{n+1} \tilde{\mathbf{q}}^{n+1}-\Delta t \sum_{k} \psi_{k}^{n} \tilde{\mathbf{F}}_{t o t, k}^{n+1} r_{k} \mathbf{n}_{k}+\Delta t \tilde{\phi}^{n+1}\left(\tilde{\mathbf{s}}^{n+1}+\mathbf{s}_{n s}^{*, n+1}\right) \Omega\right)$

Here, $\widehat{\mathbf{F}}_{t o t, k}=\widehat{\mathbf{F}}_{k}-\widehat{\mathbf{F}}_{b, k}-\widehat{\mathbf{F}}_{*, k}$. The first term of the vector $\phi^{n+1} \mathbf{q}^{n+1}$, i.e. $\phi^{n+1}\left(\bar{\eta}-z_{0}\right)^{n+1}$ expresses the volume of water inside the cell. In order to determine the individual value of $\phi^{n+1}$ and $\overline{\mathbf{q}}^{n+1}$, a corresponding water depth has to be calculated. In literature, tabulated values are used to map water volume to a certain water elevation (Panday and Huyakorn, 2004). In this work, the exact values of $\phi^{n+1}$ and $\left(\bar{\eta}-z_{0}\right)^{n+1}$ are calculated from the water volume in an iterative way. Once $\left(\bar{\eta}-z_{0}\right)^{n+1}$ is calculated, $\phi^{n+1}, q_{x}$ and $q_{y}$ can be determined. Using an iterative solution significantly increases the computational cost. In the current model implementation, the evaluation of porosities, i.e. Eqs. (16) and (17), turns out to be the most 
expensive part of the code, taking up to $15 \%$ of the total CPU time. It is important to note that this is not the one-off evaluation of porosity, but all evaluations summed up. The reason for the high cost is that, due to their dependency on water depth, the porosity values have to be evaluated several times for different water depths during one time step. Eq. (16) is solved at the beginning of the time step in each cell. During MUSCL reconstruction Eq. (17) is solved at each edge. Then, Eq. (16) is solved repeatedly during the iterative procedure to determine the new water depth and porosity in the next time step. For a two-stage Runge-Kutta method all these calculations have to be carried out twice in each time step.

A more efficient, approximate solution for this problem is presented in Yu and Lane (2006). However, in our opinion the calculation of the water depth should have very high accuracy, so the mass conservation is strictly satisfied.

The presented scheme is of explicit nature and therefore its stability is restricted by the Courant-Friedrichs-Lewy criterion (CFL), although the theoretical analyses of the stability constraint are very complicated for the present equations. The CFL criteria given in Sanders et al. (2008) is

$C r=\psi \lambda \Delta r \frac{\Delta t}{\phi \Omega} \leqslant 1$

where $\lambda=\left|u n_{x}+v n_{y}\right|+\sqrt{g h}$ is the largest wavespeed at the cell edge. Numerical experiments show that Eq. (40) degenerates the time step in cases with small porosity such that in the worst case the simulation comes to a halt.

In this work, the CFL number is heuristically calculated as

$C r=\frac{(|\mathbf{v}|+\sqrt{g h}) \Delta t}{\Delta x}$

For the presented cases, $\mathrm{Cr}<0.3$ gives satisfactory results.

\subsection{Boundary conditions}

Boundary conditions are imposed on the boundary edge of the cell according to the theory of characteristics proposed in Song et al. (2011). State variables at the boundary edge can be computed using Riemann invariants. The porosities are mirrored from the cell inside the domain.

\section{Computational examples}

Kim et al. (2015) noted three types of errors of the porous shallow water model: (1) structural model errors, (2) scale errors and (3) porosity model errors. Errors of type 1 refer to the limitations of the mathematical model concept of the shallow water equations and are defined by the difference between measurement and highresolution model (HR) results. Errors of type 2 are associated with the lack of sufficient grid resolution. In Kim et al. (2015) it is suggested to study the difference between HR model results and the HR model results which have been averaged over each porosity model grid cell (CR, standing for coarse-resolution). Errors of type 3 are the errors introduced by the porosity concept and are defined as the difference between the porosity model results (AP, standing for anisotropic porosity) and the CR model results.

Following the studies presented in Kim et al. (2015), the errors are computed using an $L_{1}$-norm:

$L_{1}=\frac{1}{N} \sum_{j=1}^{N}\left|w_{1, j}-w_{2, j}\right|$

Here, $N$ is the number of points compared, $w$ stands for a variable, e.g. $h$ or $q, w_{1, j}$ and $w_{2, j}$ are results of two different models and $j$ is the point index. The AP model is first calibrated by minimizing the $L_{1}$-norm in a manual calibration process. In a second step the fine calibration is automated using the SciPy library (Jones et al., 2001). In the following examples, the errors of type 1, 2 and 3 as well as the differences between HR model and AP model, and AP model and measurement data are presented.

The classical shallow water model used for obtaining the reference results is the model presented in Simons et al. (2014). All simulations are run in parallel with 8 threads of an Intel ${ }^{\circledR}$ Core $^{\mathrm{TM}}$ i7-2600 CPU (3.40 GHz).

All triangular meshes are generated using the mesh generator Gmsh (Geuzaine and Remacle, 2006).

\subsection{Idealized test case: dam-break flow through artificial street network}

The first test case is a test case which is initially proposed in Guinot (2012). The HR model is used to generate the reference solution. The aim of this test case is to assess the sensitivity of the porosities $\phi$ and $\psi$ to the mesh. Thus, different meshing strategies for the AP model are compared against each other. A second objective is to test the sensitivity of the model to the proposed drag coefficient $a \cdot c_{D}^{0}$. For this purpose, the drag coefficient is varied and the results are compared.

\subsubsection{Domain description, initial and boundary conditions}

The computational domain is an infinitely long, frictionless street with periodical structures as shown in Fig. 3. The initial water elevation on the left is $\eta_{L}=10 \mathrm{~m}$ and on the right side $\eta_{R}=0.25 \mathrm{~m}$. The discontinuity of water elevation located at $x=0$, which is the middle of the domain.

The HR model is two-dimensional and uses triangular cells with a characteristic length of $1 \mathrm{~m}$. The AP model is one-dimensional with a cell length of $40 \mathrm{~m}$.

\subsubsection{Influence of different meshes and areal porosity}

The AP model is expected to be sensitive to the mesh, because the areal porosity $\psi$ depends on the position of the cell edge. Two configurations are investigated: (1) the cell edge is located at the narrow section of the street network (cf. Fig. 3 (bottom left)), i.e. $\psi=1 / 7,(2)$ the cell edge is located in the wider section of the street network (cf. Fig. 3 (bottom right)), i.e. $\psi=1$. The volumetric
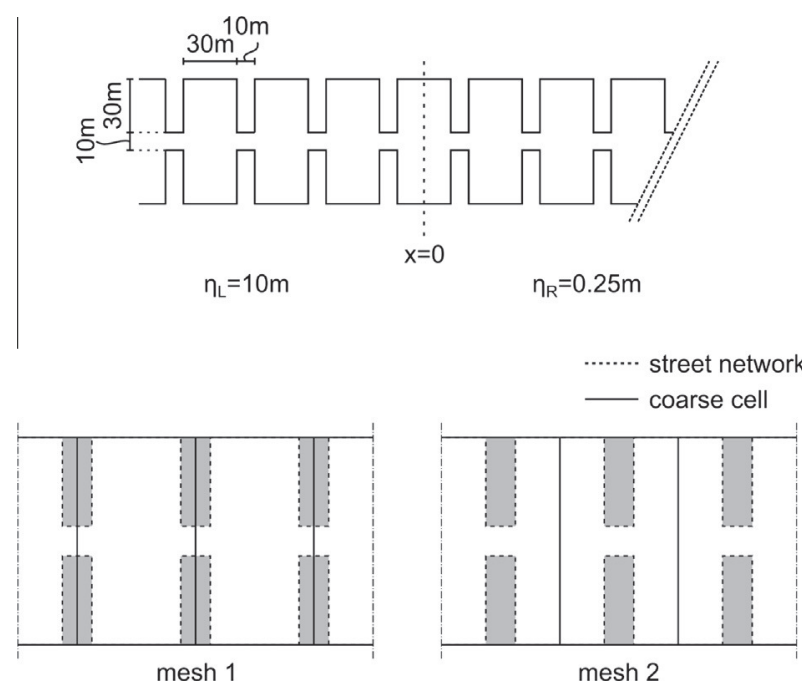

Fig. 3. Idealized test case: dam-break flow through periodic structures: top view on domain (not correctly scaled) (Guinot, 2012) (top), meshing strategies (bottom). 
porosity in both cases is the same and is calculated to be $\phi=11 / 14$. Thus, the difference in results can be directly related to the different areal porosities.

Comparison of model results at $t=50 \mathrm{~s}$ are plotted in Fig. 4 (top). The AP model with $\psi=1 / 7$ (mesh 1 ) produces the blockade effects of the structure better than the AP model with $\psi=1$ (mesh 2 ). Because both models do not resolve the street network explicitly, they can not reproduce the local fluctuations in the water elevation. In both models, the right-traveling shock wave as well as the left-traveling rarefaction wave are not captured accurately. If the edge is placed at the narrow section of the street network (mesh 1), introduces correct amount of resistance to the flow. In upstream direction, the water depth is slightly underpredicted. While the agreement is not perfect, the AP model results resemble the HR model solution. If the edge is placed at the wide section, the model is equivalent to the isotropic porosity shallow water model of Guinot and Soares-Frazão (2006) and Soares-Frazão et al. (2008). Here, the shock and rarefaction waves advance too quickly, and the AP model results are completely different from the HR model results.

The CR model is compared with the AP model with $\psi=1 / 7$ in Fig. 4 (middle left) and with the AP model with $\psi=1$ in Fig. 4 (middle right). The CR model is more diffusive than the HR model. Local water depth fluctuations are averaged out. The AP model with $\psi=1 / 7$ shows better agreement with the CR model results than the AP model with $\psi=1$.

This shows that the AP model results are very sensitive to the areal porosity $\psi$ and therefore are very sensitive to the mesh. Results indicate that the mesh should be constructed in such way that the cell edges are located on the blocking structures to capture their influence. If a structure is located completely inside a cell, its influence on the flow is only modeled by the volumetric porosity which can not model its obstruction to the flow sufficiently.

The right traveling shock wave in the AP model advances too slow. The reason for this might be that the local acceleration at narrow sections can not be taken into account by the AP model, which leads to an underestimation of the mass and momentum fluxes.

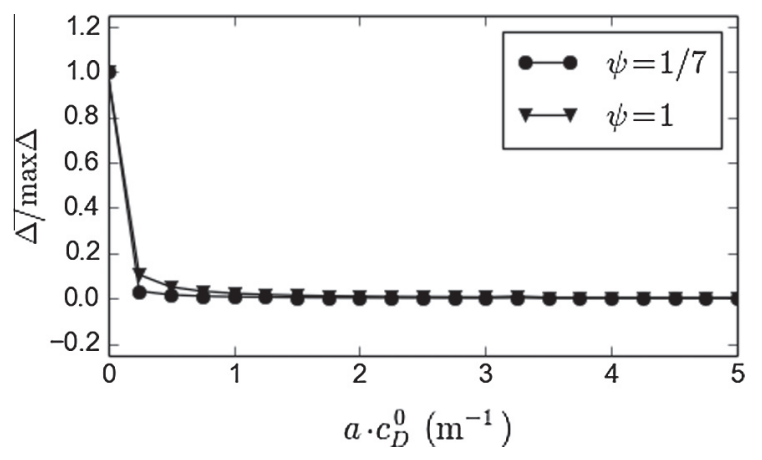

Fig. 5. Idealized test case: dam-break flow through periodic structures: sensitivity of the AP model results for different values of $a \cdot c_{D}^{0}$ at $t=50 \mathrm{~s}$ with $\Delta_{i}=L_{1}\left[A P\left(a c_{D}^{0}\right)_{i}-A P\left(a c_{D}^{0}\right)_{i+1}\right]$.
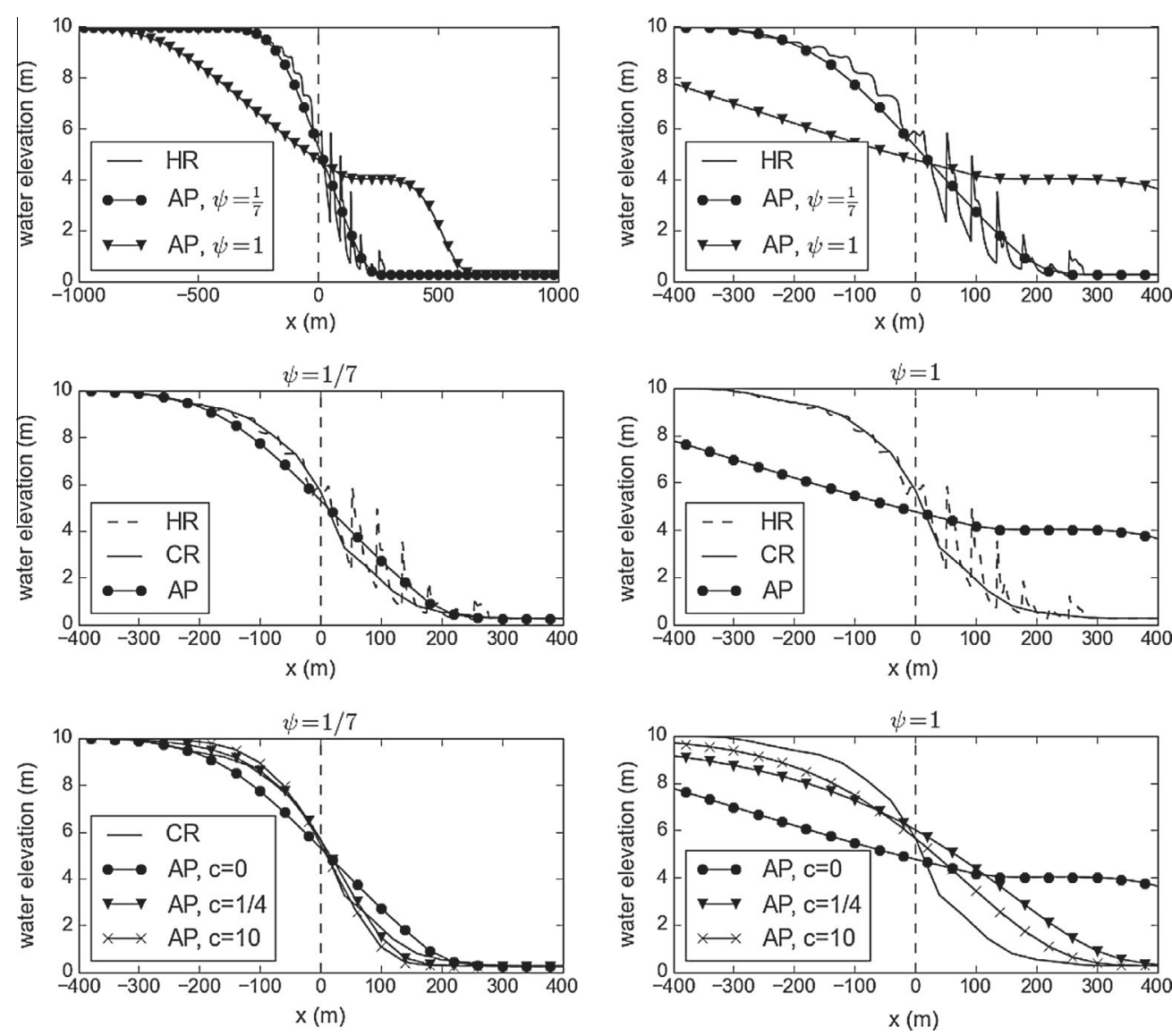

Fig. 4. Idealized test case: dam-break flow through periodic structures: results for $a \cdot c_{D}^{0}=0$ at $t=50 \mathrm{~s}$ in the whole domain (top left), detail of the results for $x=[-400,400]$ (top right), CR model results for water depth compared with HR model results and AP model with $\psi=1 / 7$ (middle left), and AP model with $\psi=1$ (middle right), CR model results for water depth compared with AP model results for different values of $c=a \cdot c_{D}^{0}$ at $t=50 \mathrm{~s}$ for $\psi=1 / 7$ (bottom left), for $\psi=1$ (bottom right). 
water elevation

- bottom elevation

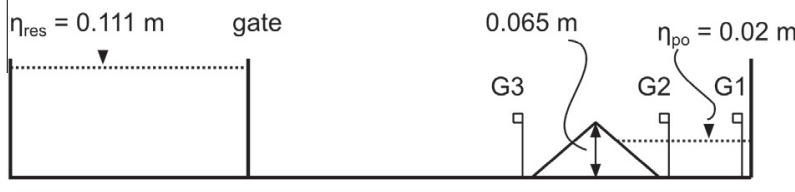

$2.39 \mathrm{~m}$

$1.61 \mathrm{~m}$

$0.9 \mathrm{~m} \longrightarrow \overrightarrow{0.7 \mathrm{~m}}$

Fig. 6. Dam-break over triangular bottom sill: side view on domain (not correctly scaled) (Soares-Frazão, 2007).
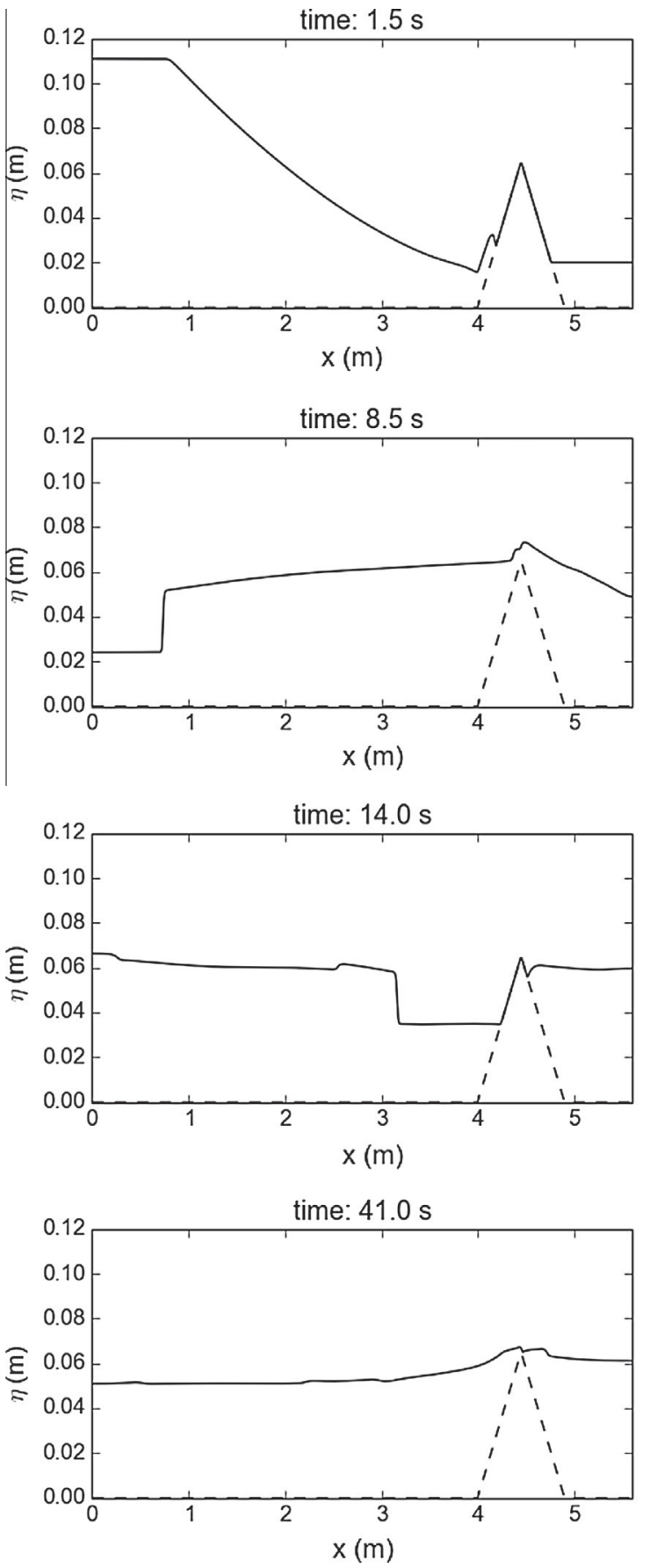

\subsubsection{Influence of drag coefficient}

The value $a \cdot c_{D}^{0}$ is now varied to study its influence on the AP model. Beginning from $a \cdot c_{D}^{0}=0$, the value is increased with a step size of $0.25 \mathrm{~m}^{-1}$ until $a \cdot c_{D}^{0}=10 \mathrm{~m}^{-1}$. Fig. 4 (bottom left) shows the AP model with $\psi=1 / 7$, while Fig. 4 (bottom right) shows the AP model results with $\psi=1$. In both cases, increasing the drag coefficient improves the agreement until a critical value $a \cdot c_{D}^{0}>1$ is exceeded. After that, the drag coefficient does not change the result anymore. For the AP model with $\psi=1 / 7$, the value $a \cdot c_{D}^{0}=0.25$ gives the best agreement. For the AP model with $\psi=1$ the agreement improves for $a \cdot c_{D}^{0}>1$ but stays overall poor.
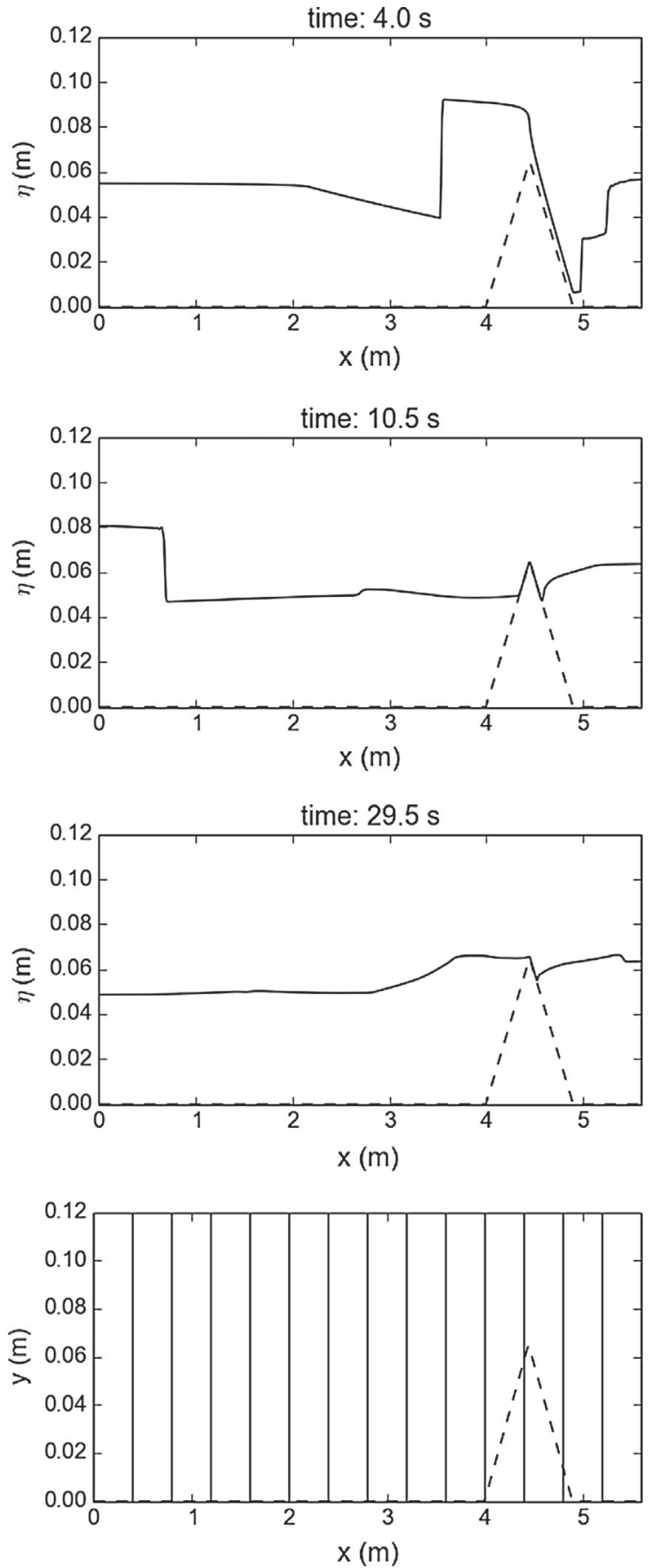

Fig. 7. Dam-break over triangular bottom sill: snapshots at different time steps of HR model results for water elevation and AP model mesh plotted over HR model bed elevation (bottom right). 
Fig. 5 compares the sensitivity of both models to the drag coefficient. For this purpose, $\Delta$ is calculated as

$\Delta_{i}=L_{1}\left(A P\left(\left(a c_{D}^{0}\right)_{i}\right), A P\left(\left(a c_{D}^{0}\right)_{i+1}\right)\right)$

where $\left(a c_{D}^{0}\right)_{0}=0,\left(a c_{D}^{0}\right)_{1}=0.25,\left(a c_{D}^{0}\right)_{2}=0.5$, and so on, and $A P(x)$ is the result of the AP model for the drag coefficient $x$. For a meaningful comparison, Fig. 5 shows a normalized value obtained by dividing each $\Delta_{i}$ by the maximum $\Delta_{i}$, i.e.

$\Delta_{n, i}=\frac{\Delta_{i}}{\max \Delta_{i}}$

Fig. 5 shows, that the AP model with $\psi=1 / 7$ is less sensitive to the drag coefficient than the AP model with $\psi=1$. This implies that the areal porosity effect dominates the flow such that the influence of the drag force on the momentum is less significant. For values $a c_{D}^{0}>1$, the influence of the increasing drag coefficient is negligible. This is because the numerical scheme limits the drag force source term in such way that the flow direction is not reversed.

If the areal porosities are large, the numerical flux is not limited as strictly and blocking effects of the obstructions are not reproduced as well as for smaller areal porosities. In this case, increasing the drag coefficient has larger influence on model results. The drag force depends only on the volumetric porosity, which is the same for both cases. Increasing the drag coefficient has a similar effect as increasing the friction coefficient and the results are similar to the findings by Liang et al. (2007) who capture the effect of buildings to some extent using an increased roughness coefficient. If the areal porosities are small, the flow is blocked more severely at the edges and the flow velocity is not as high as in the unobstructed flow. Therefore, changing the value of $a \cdot c_{D}^{0}$ does not effect the results as much.

\subsection{Dam-break flow over a triangular bottom sill}

Herein, the depth-dependent porosity is demonstrated by replicating a laboratory experiment conducted at the Universite catholique de Louvain, Belgium (Soares-Frazão, 2007).

\subsubsection{Domain description, initial and boundary conditions}

The experiment was carried out in a $5.6 \mathrm{~m}$ long and $0.5 \mathrm{~m}$ width channel. The peak of the triangular bottom sill is located at $x=4.45 \mathrm{~m}$ and is $0.065 \mathrm{~m}$ high. The sill is symmetrical and has a base length of $0.9 \mathrm{~m}$. The initial conditions and the geometry is given in Fig. 6. An initial water elevation of $\eta_{\text {res }}=0.111 \mathrm{~m}$ is ponding in the reservoir before the gate is opened. The gate is located at $x=2.39 \mathrm{~m}$. On the downstream side of the sill, water is at rest with an initial water elevation of $\eta=0.02 \mathrm{~m}$.

The HR model uses square shaped cells with a side length of $0.01 \mathrm{~m}$. It is noted that this test case is essentially onedimensional. However, the domain was discretized in two dimen-
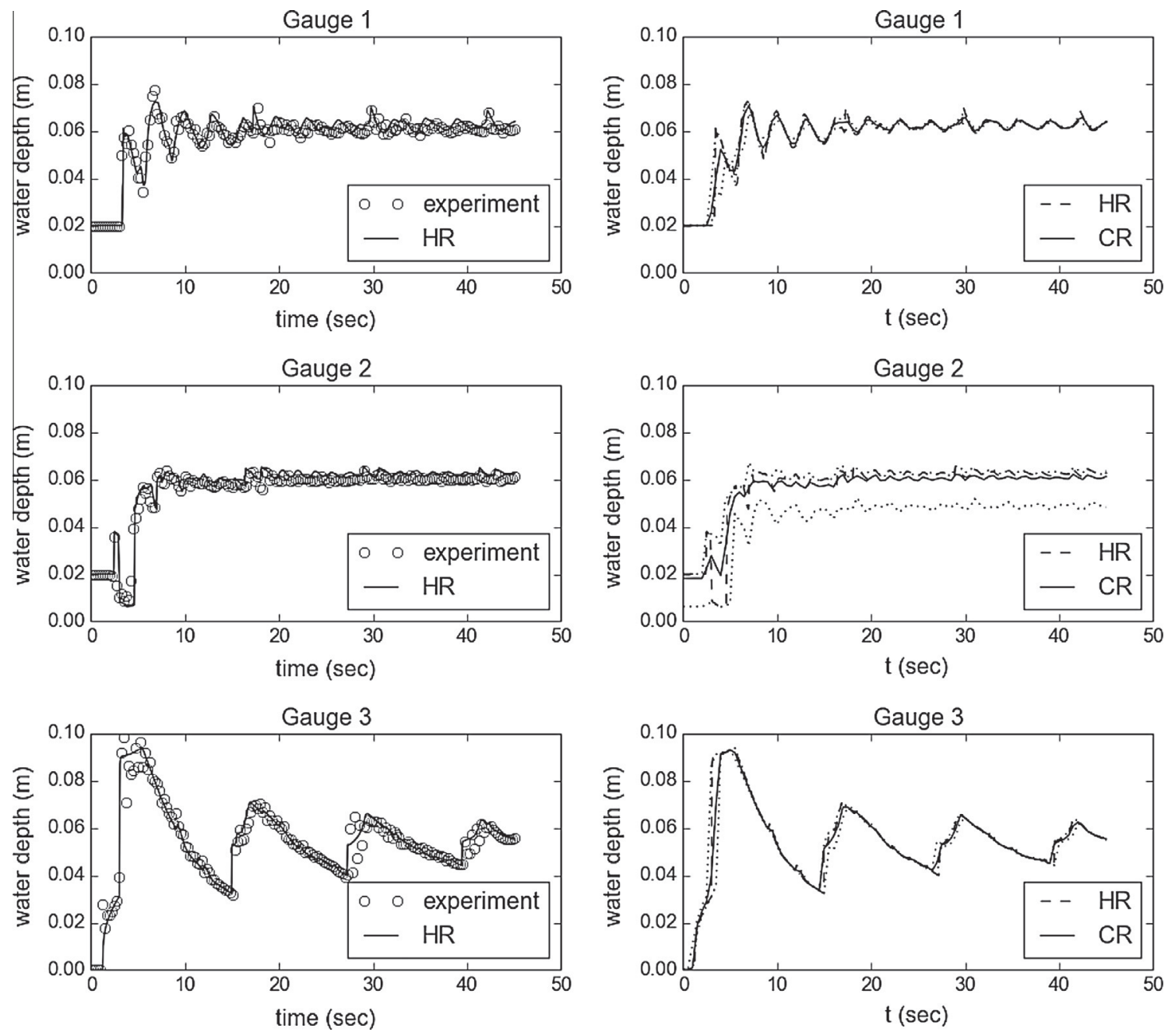

Fig. 8. Dam-break over triangular bottom sill: HR model results for water depth compared with experimental data (Soares-Frazão, 2007) (left), CR model results for water depth compared with HR model results, dotted lines denote the minimum and maximum values inside the coarse cell (right). 
sions, resulting in a mesh with 28,000 cells. The AP model uses square shaped cells with side length of $0.4 \mathrm{~m}$, which gives a mesh with 56 cells. The bottom of the AP model is completely flat and the sill is accounted for only by the porosity terms. Fig. 7 (bottom right) shows a sideview of the AP model mesh with the HR model bed elevation plotted for reference.

Measured water depth over time is available at 3 measurement gauges, located at $x=5.575 \mathrm{~m} \mathrm{(G1),} x=4.925 \mathrm{~m} \mathrm{(G2)} \mathrm{and}$ $x=3.935 \mathrm{~m}(\mathrm{G} 3)$. The locations of the gauges are given in Fig. 6 .

The roughness of the channel is quantified in Soares-Frazão (2007) with a Manning's coefficient of $n=0.011 \mathrm{~s} \mathrm{~m}^{-1 / 3}$. This value is used both in the HR and the AP model.

\subsubsection{Model calibration and run time}

The AP model is calibrated by changing the value $a \cdot c_{D}^{0}$ in Eq. (35). Calibration is carried out manually using the CR model as reference. Good agreement has been achieved with $a \cdot c_{D}^{0}=5 \mathrm{~m}^{-1}$. The HR model takes about $4000 \mathrm{~s}$ to finish, while the AP model takes only $3.5 \mathrm{~s}$. This corresponds to a speedup of about 1140 .

\subsubsection{Error analysis}

4.2.3.1. Structural model errors. This test case features an obstruction that is unsubmerged at the beginning of the simulation, completely submerged by the dam-break wave in the middle of the simulation, partially submerged towards the end of the simulation.
In Fig. 7, snapshots of the HR model results at various times are shown. The HR model shows excellent agreement with the experimental results, as seen in Fig. 8 (left), especially at gauge 2 and gauge 3 . The larger discrepancy at gauge 1 might be explained by the splashing of water in the experiment which can not be reproduced by the shallow water equations.

4.2.3.2. Scale errors. Scale errors are calculated by mapping the HR model results to a coarser grid, which in this study is the grid of the AP model. The value at a low resolution cell is determined by arithmetic averaging the values over all the high-resolution cells lying inside the low resolution cell. The $\mathrm{CR}$ model results show very good agreement with the HR model results, as seen in Fig. 8 (right), where the comparison at the three gauges is shown. The dotted lines show the maximum and minimum water depths sampled inside the coarse grid. It can be seen that at gauge 1 and gauge 3 , the difference between the minimum and the maximum water depth is low. At gauge 2, which is located just behind the sill, the deviation is high. Owing to the reflected waves, the flow at gauge 2 is more complex than at the other gauges. Consequently, here the agreement between CR model and HR model is not as close as at the other gauges. It is observed that the CR model introduces some diffusion to the results and the curves are smoother than the HR model results.
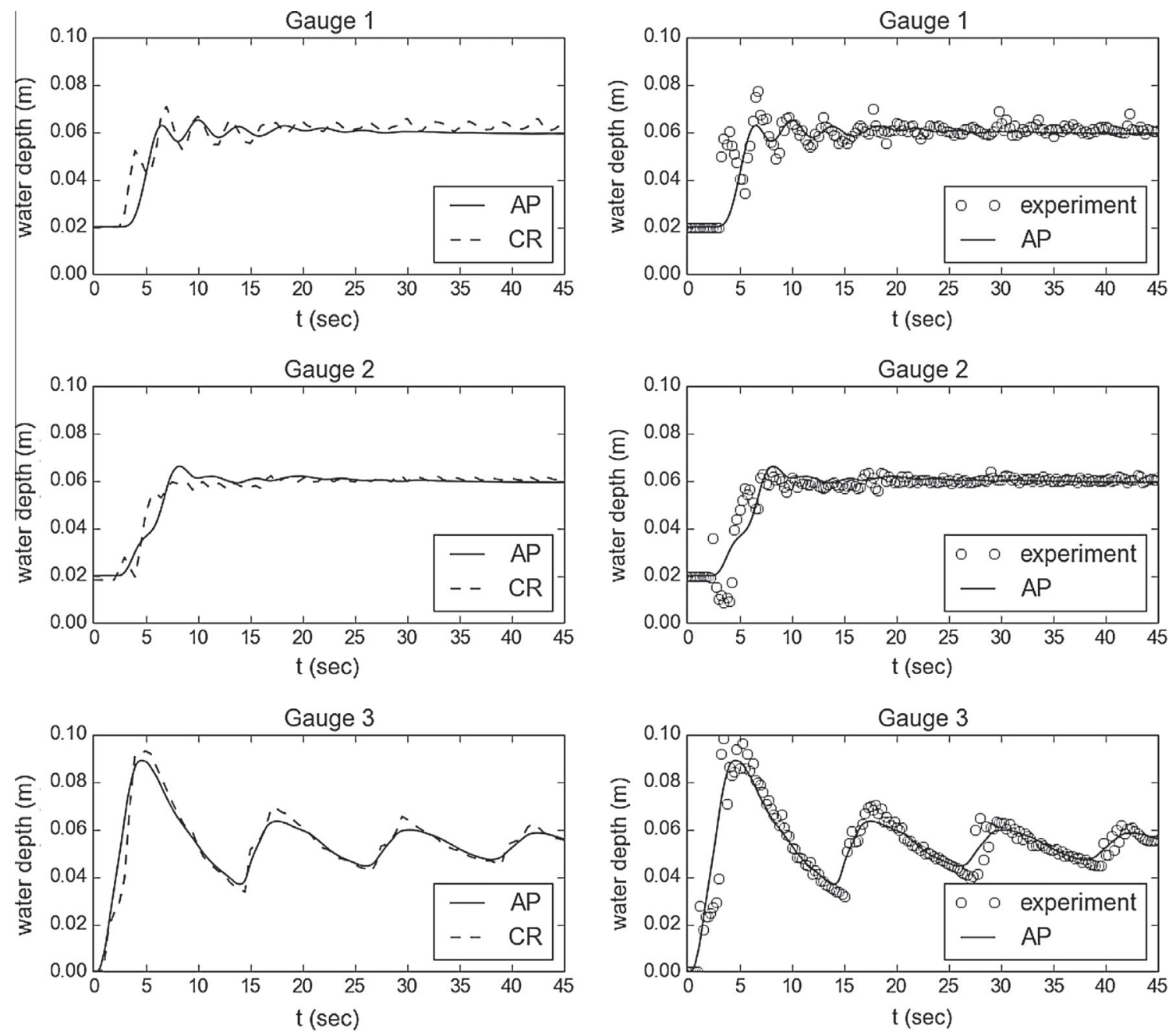

Fig. 9. Dam-break over triangular bottom sill: AP model results for water depth compared with CR model results (left), AP model results for water depth compared with experimental data (Soares-Frazão, 2007) (right). 
4.2.3.3. Porosity model errors. The porosity model errors are assessed by comparing AP model results to CR model results, as shown in Fig. 9 (left). The AP model shows good agreement with the CR model at all gauges. At gauge 1, which is located furthest away from the gate the predicted wave arrives a bit late. However, after $5 \mathrm{~s}$ the arrival time of the second peak is captured despite the slightly undershot peak water level. The third peak is captured accurately. After that, the AP model does not predict as much fluctuation as the $C R$ model but the average water elevation does not differ much. The agreement at gauge 2 and gauge 3 is much better. Especially at gauge 3 all waves are captured with good agreement. At gauge 2, the rise of the curve starts correctly but the AP model overshoots the CR model at about $8 \mathrm{~s}$. A comparison between AP model result with experimental data is shown in Fig. 9 (right). The AP model reproduces the experimental data well.

\section{Table 1}

Dam-break over triangular bottom sill: summary of shallow water model formulations and corresponding meshes (HR: High-resolution, CR: averaged HR model, AP: anisotropic porosity); $L_{1}$-norm is calculated with regard to the experimental results.

\begin{tabular}{llllll}
\hline Model & Mesh type & Cell size $(\mathrm{m})$ & Cell nr. & Time $(\mathrm{s})$ & $L_{1}(\mathrm{~m})$ \\
\hline HR & Square & 0.01 & 28000 & 4000 & 0.0024 \\
CR & Square & 0.01 & 28000 & 4000 & 0.0031 \\
AP & Square & 0.4 & 56 & 3.5 & 0.0035 \\
\hline
\end{tabular}

Table 2

Dam-break over triangular sill: model error $\left(E_{1}\right)$, scale error $\left(E_{2}\right)$ and porosity error $\left(E_{3}\right)$.

\begin{tabular}{ll}
\hline Type & $L_{1}(\mathrm{~m})$ \\
\hline$E_{1}$ & 0.0024 \\
$E_{2}$ & 0.0016 \\
$E_{3}$ & 0.0038 \\
\hline
\end{tabular}

4.2.3.4. Summary. The $L_{1}$-errors are listed in Tables 1 and 2. In both tables, the errors are calculated as the arithmetic mean of the errors at the 3 gauges. Table 1 shows a summary of the cell sizes and $L_{1}$-errors for HR model, CR model and AP model. Here, the errors are calculated using the experimental data as a reference. Overall, the errors are two orders of magnitude smaller than the initial water elevation in the reservoir $\left(\eta_{\text {res }}=0.111 \mathrm{~m}\right)$. The $L_{1}$ errors for structural, scale and porosity model errors are summarized in Table 2. All errors are in the same order of magnitude, which is one order of magnitude smaller than the maximum measured water depth. The porosity model $\left(E_{3}\right)$ error is the largest, followed by the structural model error $\left(E_{1}\right)$. The scale error $\left(E_{2}\right)$ is the smallest error. It is concluded that in this example, the error introduced by the coarse grid is the smallest. The mathematical model limitation of the shallow water equations introduces larger errors than the grid coarsening, but the largest error is introduced by not resolving the sill explicitly.

\subsection{Dam-break flow through an idealized city}

In this computational example, results of a dam-break experiment conducted at the Université catholique de Louvain, Belgium (Soares-Frazão and Zech, 2008) are numerically reproduced.

\subsubsection{Domain description, initial and boundary conditions}

The domain is a $35.8 \mathrm{~m}$ long and $3.6 \mathrm{~m}$ wide channel with horizontal bed. The idealized city consists of $5 \times 5$ buildings, each of them being a square block with a side length of $0.30 \mathrm{~m}$. The distance between the blocks is $0.10 \mathrm{~m}$. The center of the building block is placed $5.95 \mathrm{~m}$ away from the gate and rotated $22.5^{\circ}$ in counter-clockwise direction around its center. The dam-break is constructed by opening a $1 \mathrm{~m}$ gate, which initially seperates the reservoir, where water is ponding at $0.40 \mathrm{~m}$, from the rest of the channel, where a very thin layer of $0.011 \mathrm{~m}$ water is reported.
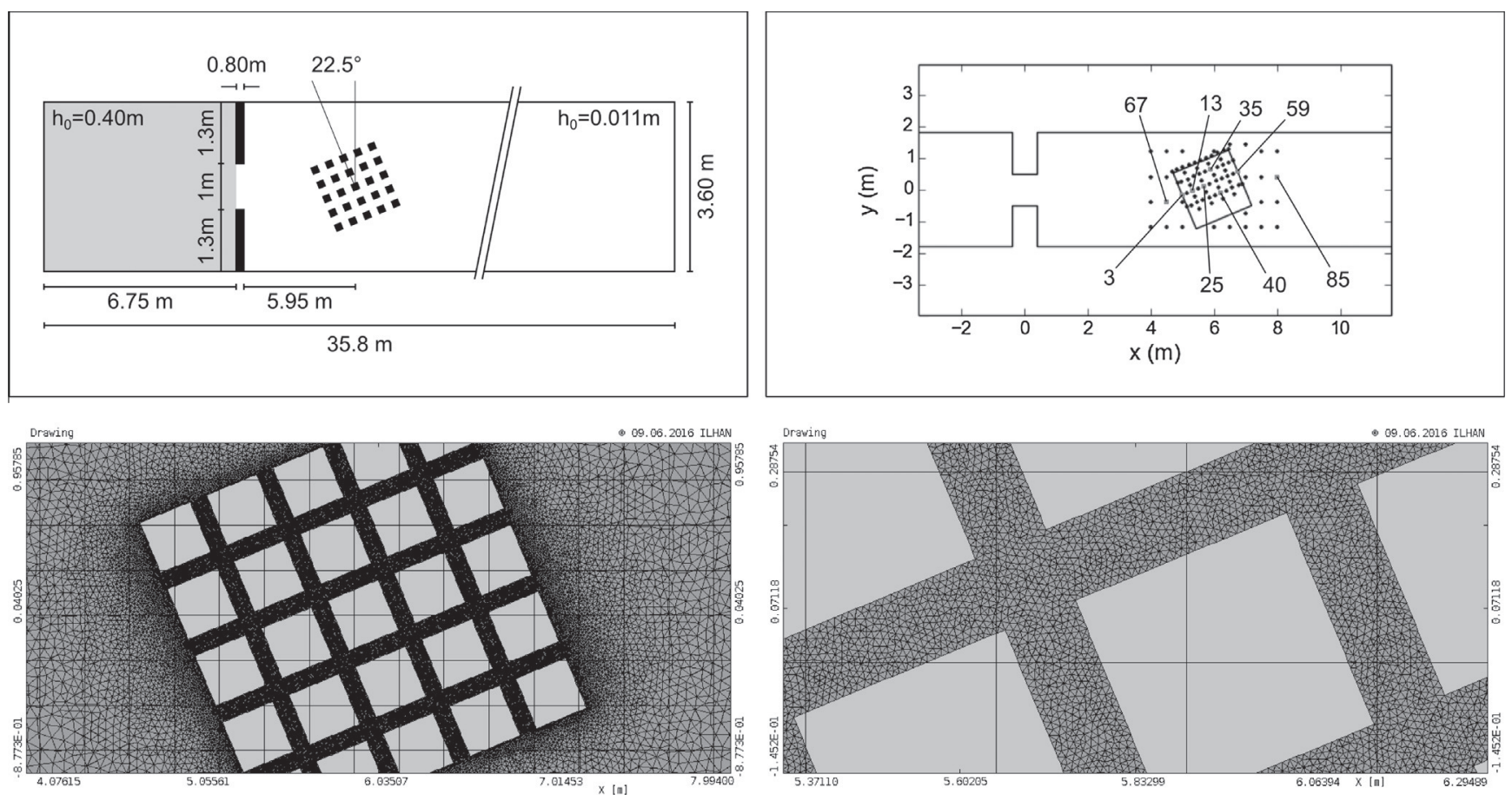

Fig. 10. Dam-break through idealized city: top view on domain (not correctly scaled) (Soares-Frazão and Zech, 2008) (top left), position of all 87 gauges (black), results are plotted for 8 gauges (indicated by their numbers), the boundary of the building block is plotted for reference (top right), comparison of HR model mesh (triangular) and CR and AP model mesh (square), meshing of the building block (bottom left), mesh detail between houses (bottom right). 
For further details on the experimental setup and employed measurement techniques, the reader is referred to Soares-Frazão and Zech (2008). The domain is illustrated in Fig. 10 (top left), where the reservoir is colored in gray.

The computational domain only includes the reservoir and the first $16 \mathrm{~m}$ of the channel. For the duration of the simulations, $t=15.5 \mathrm{~s}$, the shock wave does not travel further than this length. The downstream boundary is an open boundary and all other boundaries are closed boundaries.

The HR model uses a triangular mesh with variable cell sizes: the reservoir is discretized with cells with a characteristic length of $l_{c, 1}=0.3 \mathrm{~m}$. The area inside the channel which is sufficiently far away from the building blocks is discretized with a characteris- tic length of $l_{c, 2}=0.1 \mathrm{~m}$. The space between the buildings is discretized with a characteristic length of $l_{c, 3}=0.01 \mathrm{~m}$. The buildings are represented as holes in the mesh, which is a method commonly used in urban flood modeling (Schubert et al., 2008). Hence, the gap between two buildings is discretized with about 10 cells and the total cell number is 96339 . The AP model uses squareshaped cells with side length $0.25 \mathrm{~m}$, whereby the volumetric porosity is calculated using 125 subgrid cells, resulting in a mesh with 1272 cells. The HR mesh is compared to the AP model mesh in Fig. 10 (bottom). Both meshes in the region of the building block is shown in Fig. 10 (bottom left), while in Fig. 10 (bottom right) a close-up view is shown. A building is in general contained in 4 AP model cells. The buildings do not align with the cell edges. As
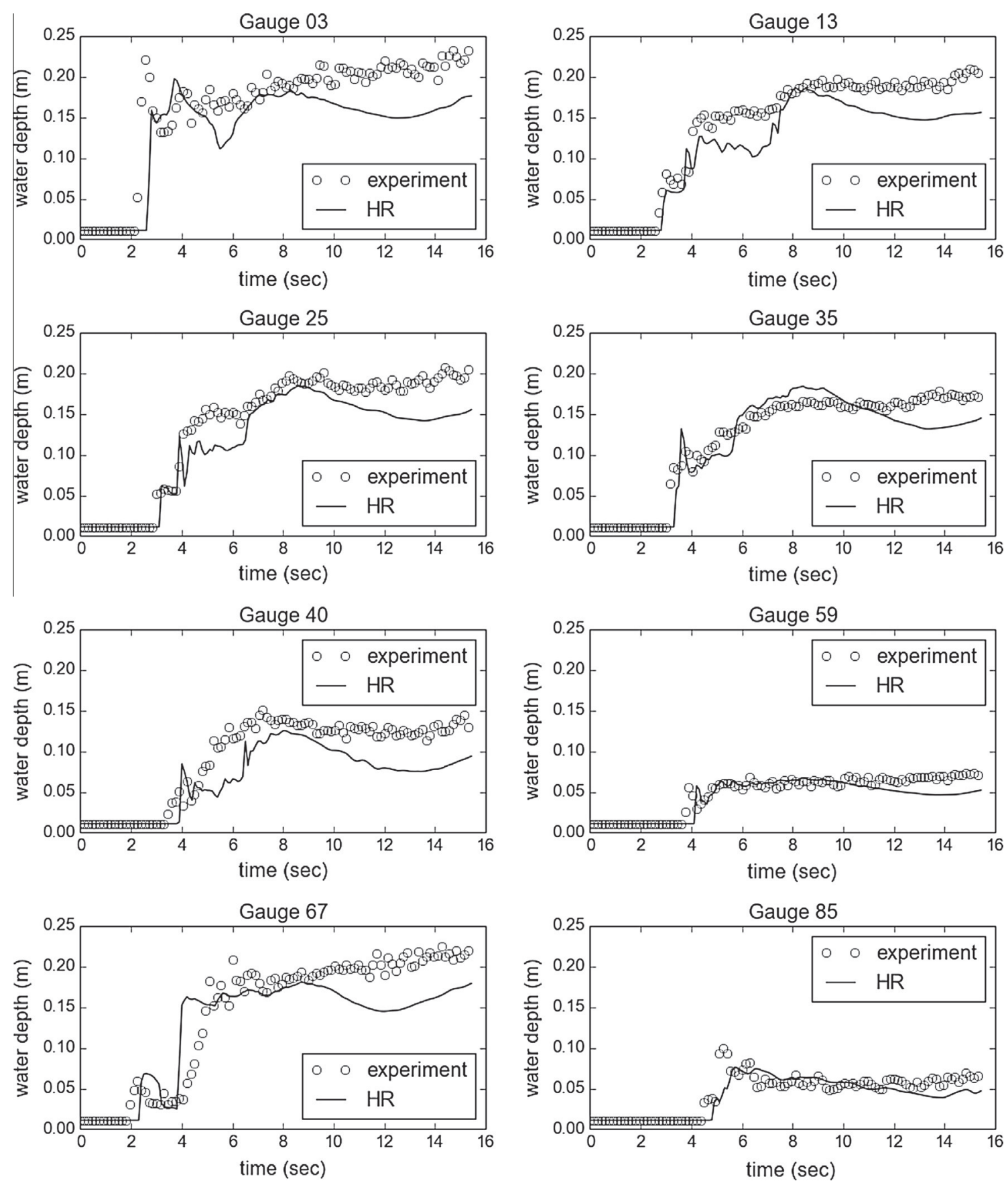

Fig. 11. Dam-break through idealized city: HR model results for water depth compared with experimental data of Soares-Frazão and Zech (2008). 
discussed in Section 4.1, the blocking effect of buildings is not captured accurately if the building is positioned inside the cell instead of at the edge, but this is inevitable for some fron-row houses (cf. Fig. 10 (bottom)).

Experimental data are available at 87 measurement gauges distributed inside the channel (Soares-Frazão and Zech, 2008). The positions of these gauges are given in Fig. 10 (top right). In the discussion, results are plotted for 8 gauges, namely gauges $3,13,25$, 35, 40, 59, 67 and 85 .

The roughness of the channel has been estimated in SoaresFrazão and Zech (2008) with a Manning's coefficient of $n=0.01 \mathrm{~s} \mathrm{~m}^{1 / 3}$. This value is used for both the HR and the AP model.
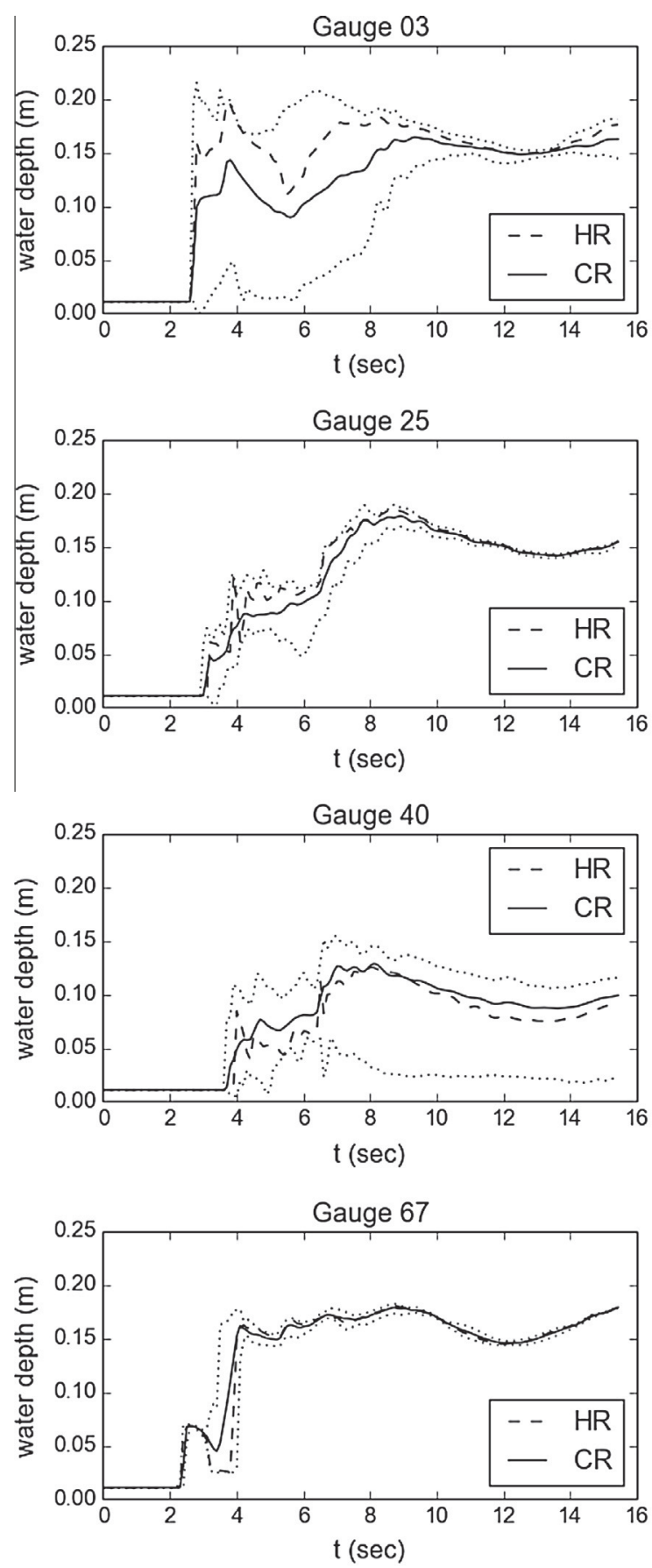

\subsubsection{Model calibration and run time}

The AP model is calibrated with the value $a \cdot c_{D}^{0}$ in the drag law, given in Eq. (35). Calibration is carried out with regard to the $C R$ model results using Brent's algorithm for minimization (Brent, 1973). Brent's search returns $a \cdot c_{D}^{0}=1.9 \mathrm{~m}^{-1}$ with a corresponding $L_{1}$-error of $0.025 \mathrm{~m}$. The HR model simulation takes about $3000 \mathrm{~s}$ to finish. The AP model requires about $4 \mathrm{~s}$. Consequently, the speedup is calculated as 750 .

\subsubsection{Error analysis}

4.3.3.1. Structural model errors. The HR model makes overall an acceptable prediction of the water depth at the evaluated gauges. In Fig. 11, the water depth calculated by the HR model at the
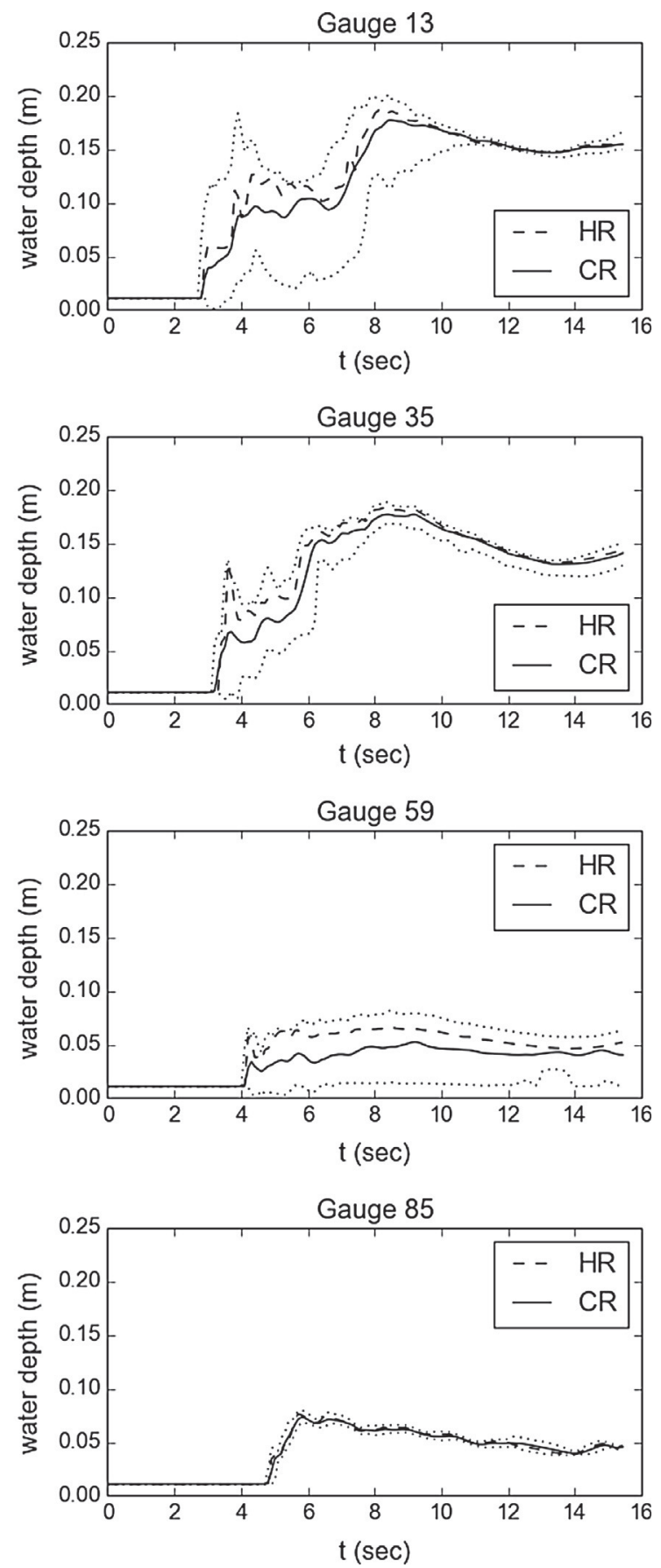

Fig. 12. Dam-break through idealized city: CR model results for water depth compared with HR model results, dotted lines denote the minimum and maximum values inside the coarse cell. 

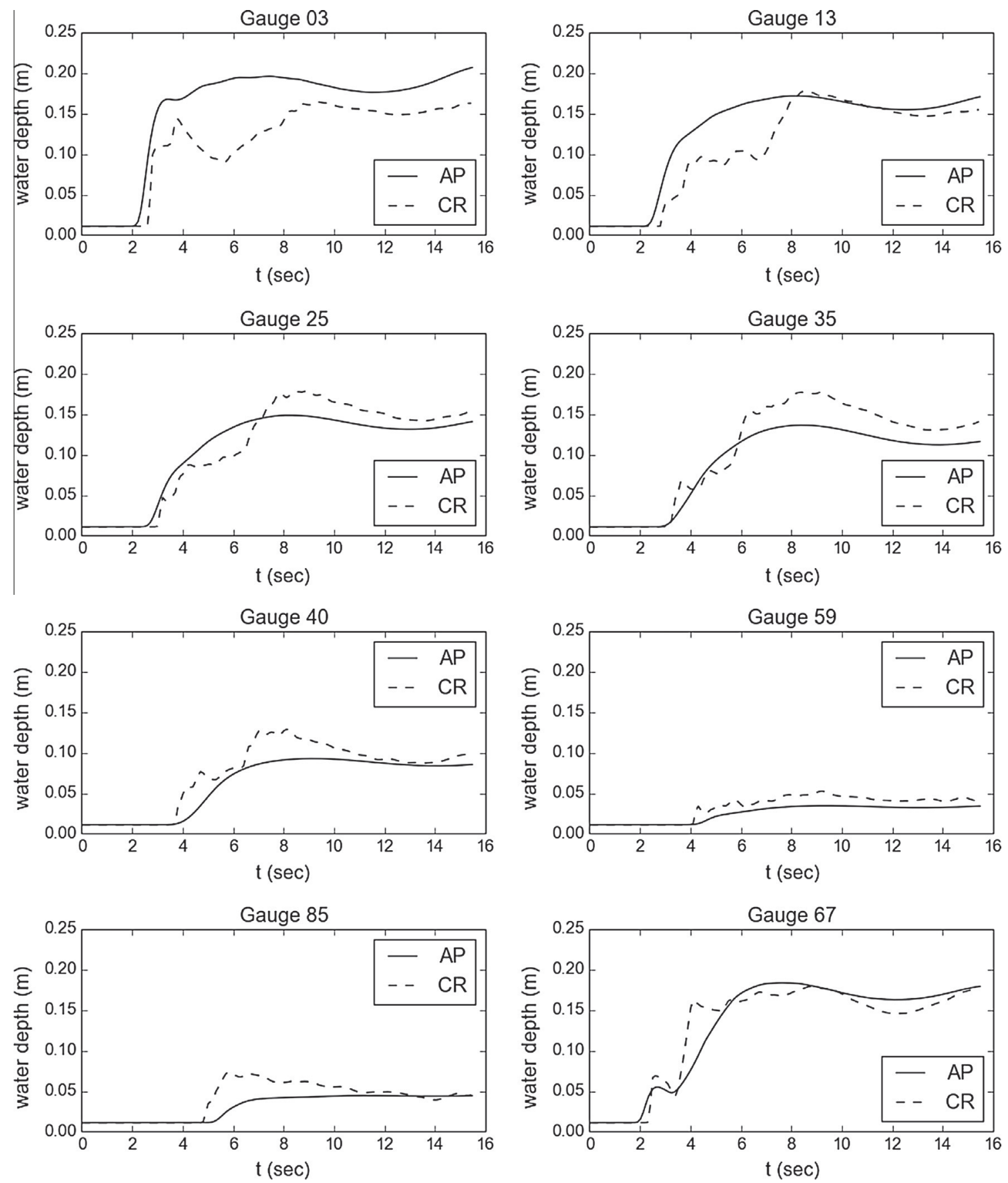

Fig. 13. Dam-break through idealized city: AP model results for water depth compared with CR model results.

Table 3

Dam-break through idealized city: summary of shallow water model formulations and corresponding meshes (HR: High-resolution, CR: averaged HR model, AP: anisotropic porosity); $L_{1}$-norm is calculated with regard to the experimental results.

\begin{tabular}{llllll}
\hline Model & Mesh type & Cell size $(\mathrm{m})$ & Cell nr. & Time $(\mathrm{s})$ & $L_{1}(\mathrm{~m})$ \\
\hline HR & Triangular & $0.01-0.3$ & 95975 & 3000 & 0.020 \\
CR & Triangular & $0.01-0.3$ & 95975 & 3000 & 0.021 \\
AP & Square & 0.25 & 1272 & 4 & 0.026 \\
\hline
\end{tabular}

aforementioned gauges is plotted together with the measured water depth. The arrival time of the wave is predicted correctly at all gauges, although the HR model predicts a slightly later
Table 4

Dam-break through idealized city: model error $\left(E_{1}\right)$, scale error $\left(E_{2}\right)$ and porosity error $\left(E_{3}\right)$.

\begin{tabular}{ll}
\hline Type & $L_{1}(\mathrm{~m})$ \\
\hline$E_{1}$ & 0.020 \\
$E_{2}$ & 0.018 \\
$E_{3}$ & 0.025 \\
\hline
\end{tabular}

arrival. Larger deviations between the results occur at the later stages of the simulation, where the HR model results undershoot the experimental data. For this test case, Soares-Frazão and Zech 
(2008) report lower computed water depths as well. The deviations might partly be caused by the frictionless wall-boundaries imposed at the buildings and the wave reflections that can not be modeled by the shallow water equations. The model overestimates the flow velocities, leading to overall lower water depths. As time passes, this effect becomes more significant. Gauge 67 is located in front of the houses. Overall, the characteristics of the experimental data set are captured by the HR model, i.e. the small peak at around $t=2 \mathrm{~s}$ and the rise at around $t=4 \mathrm{~s}$, however the first peak is delayed and the second rise at $t=4 \mathrm{~s}$ is too early. In general, the HR model appears to overpredict the steepness of the water level variations. This is especially distinct at the sharp rise of the HR model curve at $t=4 \mathrm{~s}$ in comparison to the smoother rise of the experimental curve. As in Soares-Frazão and Zech (2008), this indicates that the entrance contraction can not be reproduced by the mathematical model. This is also indicated by the discrepancies at gauge 3 , which is located at the entrance of the building block. The rise of the water level is again delayed. The drop in water depth at around $t=6 \mathrm{~s}$ is not observed in the experiment. Gauge 13 , located slightly behind gauge 3 , shows good agreement. Here, the front of the wave is captured accurately in time. The agreement at gauges 25, 35 and 59, which are all located between the buildings, is very well.

Gauge 40, which is also located between the buildings, shows worse agreement than the aforementioned gauges. As at gauge 3 , the general shape of the experimental data is reproduced. Finally,
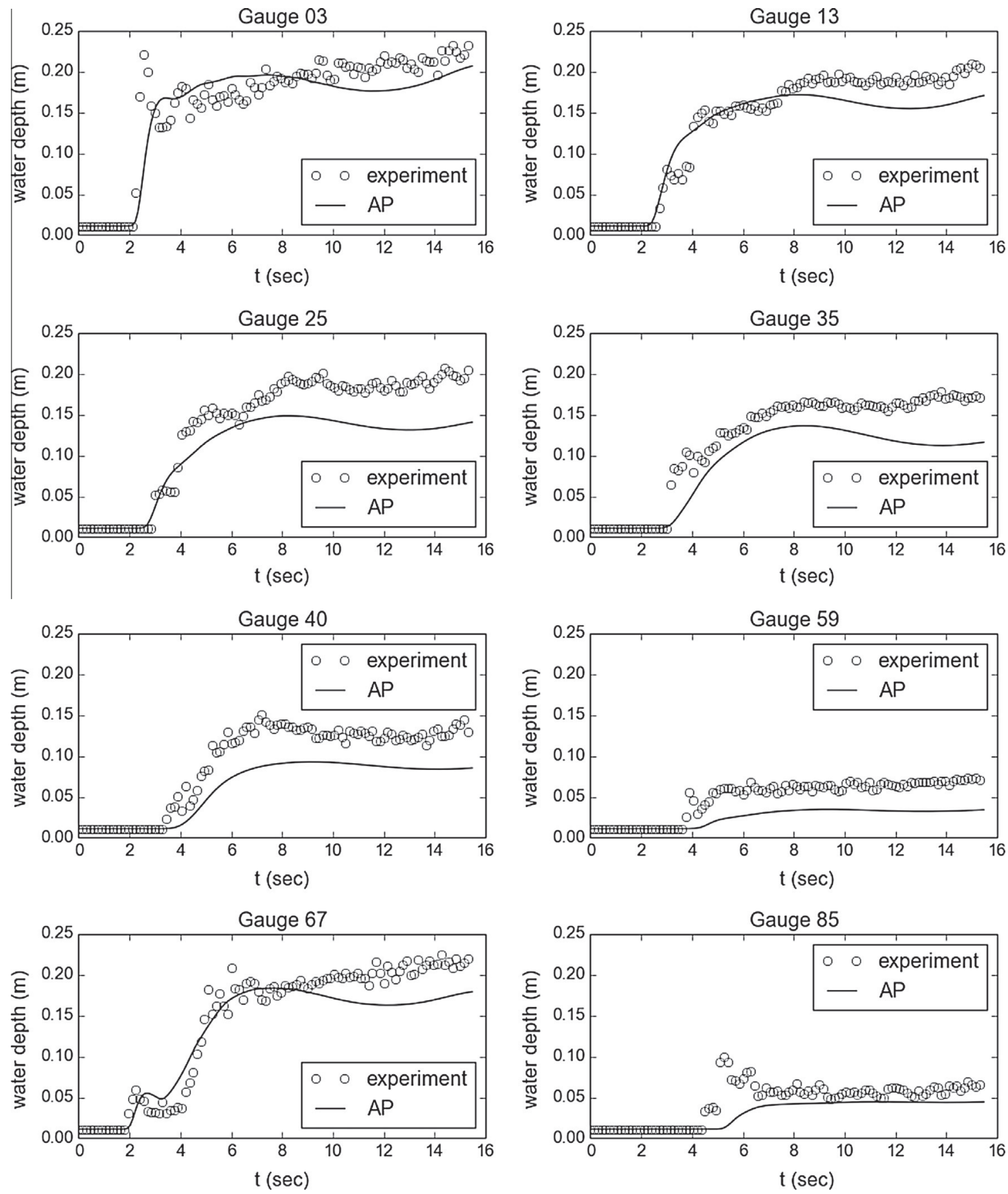

Fig. 14. Dam-break through idealized city: AP model results for water depth compared with experimental data of Soares-Frazão and Zech (2008). 
at gauge 85 , which is outside of the building block, good agreement is achieved.

Overall, this is a challenging test case for the mathematical model. The angled position of the buildings that are not aligned with the flow direction coupled with the hydraulic jump at the entrance of the building block increases the difficulty. In addition, wave reflections and turbulent eddies are not accounted for in the model. Consequently, the structural model error is relatively high.

4.3.3.2. Scale errors. In Fig. 12, the averaged water depth is plotted against the HR model water depth at the four gauges. The measured water depth is omitted to avoid cluttering the figure. Maximum and minimum values of the high-resolution cells lying inside the low-resolution cell are plotted as well. Overall, the averaging process smooths out the HR model results. Local fluctuations are not captured by the $\mathrm{CR}$ model. It is noted that a large difference between the minimum and the maximum in a coarse cell indicates complex flows. As expected, the location of the gauge can be related to the complexity of the flow. Gauges 67 and 85 are located outside of the building block and the minimum and maximum of the values at these gauges do not differ much. Conversely for the other gauges located between the buildings, the local fluctuation is high. In general, the difference between the minimum and maximum gives a good indication for the difference between HR and CR model. If the flow in a coarse cell is complex, there exist high differences between minimum and maximum water levels inside the cell. This complex flow can not be resolved on the scale of the CR model, thus it introduces an error due to scale to the CR model result. Consequently, the difference between HR and CR model is high at, e.g. Gauge 3, positioned at the front of the building block where the flow is complex, and at Gauge 40, located at a crossroad. In contrast, if the flow inside a coarse cell is relatively smooth, the loss of information due to low resolution is not that severe. This is seen, e.g. at Gauge 85, located outside of the building block.

4.3.3.3. Porosity model errors. The AP model shows acceptable agreement with the CR model, although some gauges observe less good agreement, e.g. gauge 85 the agreement is poor. In general, the results of the AP model are smoother and more "smeared" than the CR model results. In Fig. 13, AP and CR model results are plotted for eight gauges. The AP model water depth at gauge 3 shows similarities to the maximum value at this gauge. Gauges 13, 25 and 67 show good agreement. At gauge 35, the shape of the curve is reproduced but the AP model underestimates the water depth. Gauge 85, which is located behind the building block, shows the worst agreement among the eight presented gauges. The AP model is unable to reproduce the $\mathrm{CR}$ model result, with underestimated peak water level and delayed arrival time. Overall, the general properties of the AP model results, i.e. the lack of local and spatial fluctutations, are consistent with the findings in Kim et al. (2015).

4.3.3.4. Summary. An overview of the results of this computational study is given in Table 3 and 4 . The $L_{1}$-errors in Table 3 are calculated by taking the measured data by averaging the $L_{1}$-errors of all 87 gauges. Moreover, the AP model results are plotted against the measurement data in Fig. 14. The errors are as expected: the HR model has the lowest error, the CR model comes second and the AP model shows the largest error. However, the errors have the same order of magnitude and are one order of magnitude smaller than the initial water depth in the reservoir $\left(h_{0}=0.4 \mathrm{~m}\right)$. Table 4 shows the structural, scale and porosity errors $E_{1}, E_{2}$ and $E_{3}$, respectively. The values are again averaged over 87 gauges. In this example, the error due to coarser cells is smaller than the structural and porosity errors. Indeed, the CR model results show good agreement with the HR model (cf. Fig. 12), while the difference between CR model and AP model is larger.

\subsection{Rainfall-runoff in an idealized urban catchment}

A series of experiments regarding pluvial flooding in urban catchments were carried out at the Universidad de A Coruna, Spain (Cea et al., 2010). One of these experiments is studied in this computational example.

\subsubsection{Domain description, initial and boundary conditions}

Constant rainfall with an intensity of $i=300 \mathrm{~mm} / \mathrm{h}$ is applied for $20 \mathrm{~s}$ to a $2.5 \mathrm{~m}$ long and $2 \mathrm{~m}$ wide rectangular inclined domain with a slope of 0.05 . Inside of the domain, a simplified urban district is built using $0.30 \mathrm{~m} \times 0.20 \mathrm{~m}$ wooden blocks as houses. The configuration of the houses is plotted in Fig. 15 (top). The domain is initially dry. Further details regarding the experimental setup and more building configurations can be found in Cea et al. (2010). In the numerical models, the outlet of the domain is an open boundary and all other boundaries are closed. The simulation runs for $150 \mathrm{~s}$.

The HR model discretises the domain with a triangular mesh with varying cell size, starting at $l_{c, 1}=0.05 \mathrm{~m}$ at the boundary of the domain to $l_{c, 2}=0.01 \mathrm{~m}$ between the buildings, which are again
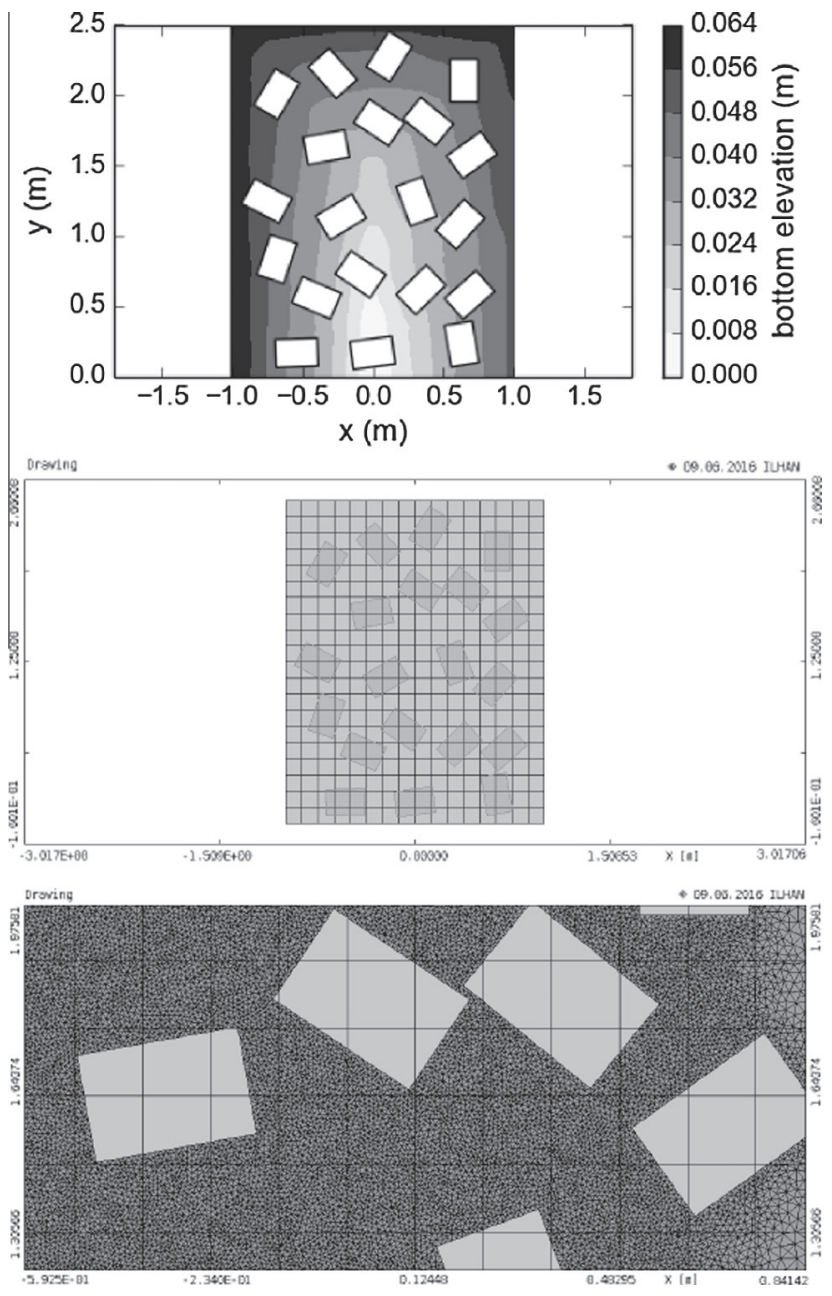

Fig. 15. Rainfall-runoff in an idealized urban catchment: bottom elevation in the domain and configuration of houses (top), CR and AP model mesh of the whole domain (middle), comparison of HR model mesh (triangular) and CR and AP model mesh (square) between houses (bottom). 
represented as holes in the mesh. The resulting mesh has 62058 cells. The AP model uses square shaped cells with a side length of $0.125 \mathrm{~m}$, which results in a mesh with 320 cells. The two meshes are compared in Fig. 15. The whole domain is plotted in Fig. 15 (middle) with the houses marked out as reference and in Fig. 15 (bottom) the region between houses. One building can be contained in approximately 6 AP model cells. Again, the alignment of the buildings does not match the AP model mesh cells.

In contrast to the previous examples, no measurement data inside the domain is available, Cea et al. (2010) measured the total discharge at the outlet of the domain.

\subsubsection{Model calibration and run time}

The roughness of the domain is reported in Cea et al. (2010) in form of a Manning's coefficient of $0.016 \mathrm{~s} \mathrm{~m}^{-1 / 3}$. The results of the HR model agree well with the experimental data, thus no further calibration is required. The HR simulation takes about $5340 \mathrm{~s}$. The AP model uses the same roughness coefficient $\left(0.016 \mathrm{~s} \mathrm{~m}^{-1 / 3}\right)$ and a drag force with $a \cdot c_{D}^{0}=0.5 \mathrm{~m}^{-1}$ (determined with Brent's method). In each cell, 400 subgrid-cells are used to calculate the porosity. The AP model simulation runs for about $43 \mathrm{~s}$, which is a speedup of about 124. The lower speedup in comparison to the first test case is because the stability criterion has to be set to $\mathrm{Cr}=0.1$ in this example. The numerical simulation of rainfall is prone to instabilities because of small water depths and the presence of the mass source (Murillo et al., 2007).

\subsubsection{Error analysis}

4.4.3.1. Structural model errors. The HR model shows good agreement with the experimental data. The discharge at the outlet of the domain as calculated by the HR model is plotted against the measured discharge in Fig. 16 (top left). In the first $10 \mathrm{~s}$ of the simulation, the model discharge overshoots the measured discharge. This has been also observed in Cea et al. (2010), and is most likely because at the beginning of the experiment the shear stress on the thin water film in the domain is holding the water back. This can not be reproduced by the shallow water model. After the first $10 \mathrm{~s}$, both hydrographs show very good agreement.

4.4.3.2. Scale errors. The CR model agrees with the HR model, yet the agreement is not as good as in the first test case, especially at the beginning of the simulation. In Fig. 16 (top right), the maximum and minimum values of the subgrid-cells are also plotted. It is seen that the peak of the curve of maximum values is about 3 times larger than the peak of the CR model while the curve of minimum values is close to zero. Generally, it can be concluded that the scale error underestimates the retention effect of the domain.

4.4.3.3. Porosity model errors. The AP model results are plotted against the CR model results in Fig. 16 (bottom left) and against the experimental results in Fig. 16 (bottom right). The AP model results show a similar evolution as the $\mathrm{CR}$ model results. The major difference between both curves is at the beginning of the simulation. The AP model undershoots the CR model results. Yet, as can be seen in Fig. 16 (bottom right), it better matches the measured discharge at the end of the domain. Fig. 17 shows a sensitivity analysis with regard to the subgrid-cell number, from which it is concluded that the model is sensitive to the subgrid-cell number.

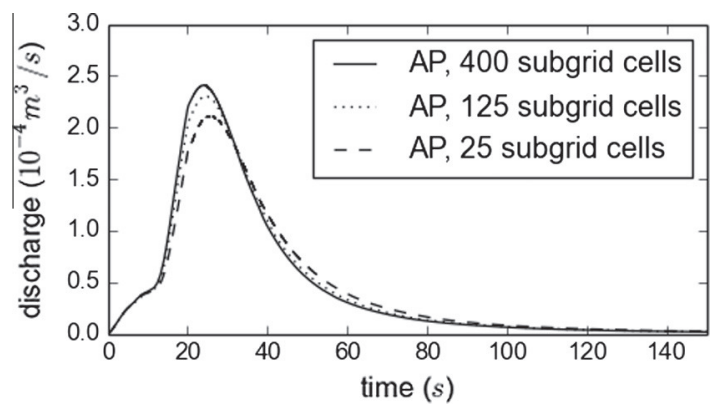

Fig. 17. Rainfall-runoff in an idealized urban catchment: Sensitivity of the subgridcell number on the AP model results.
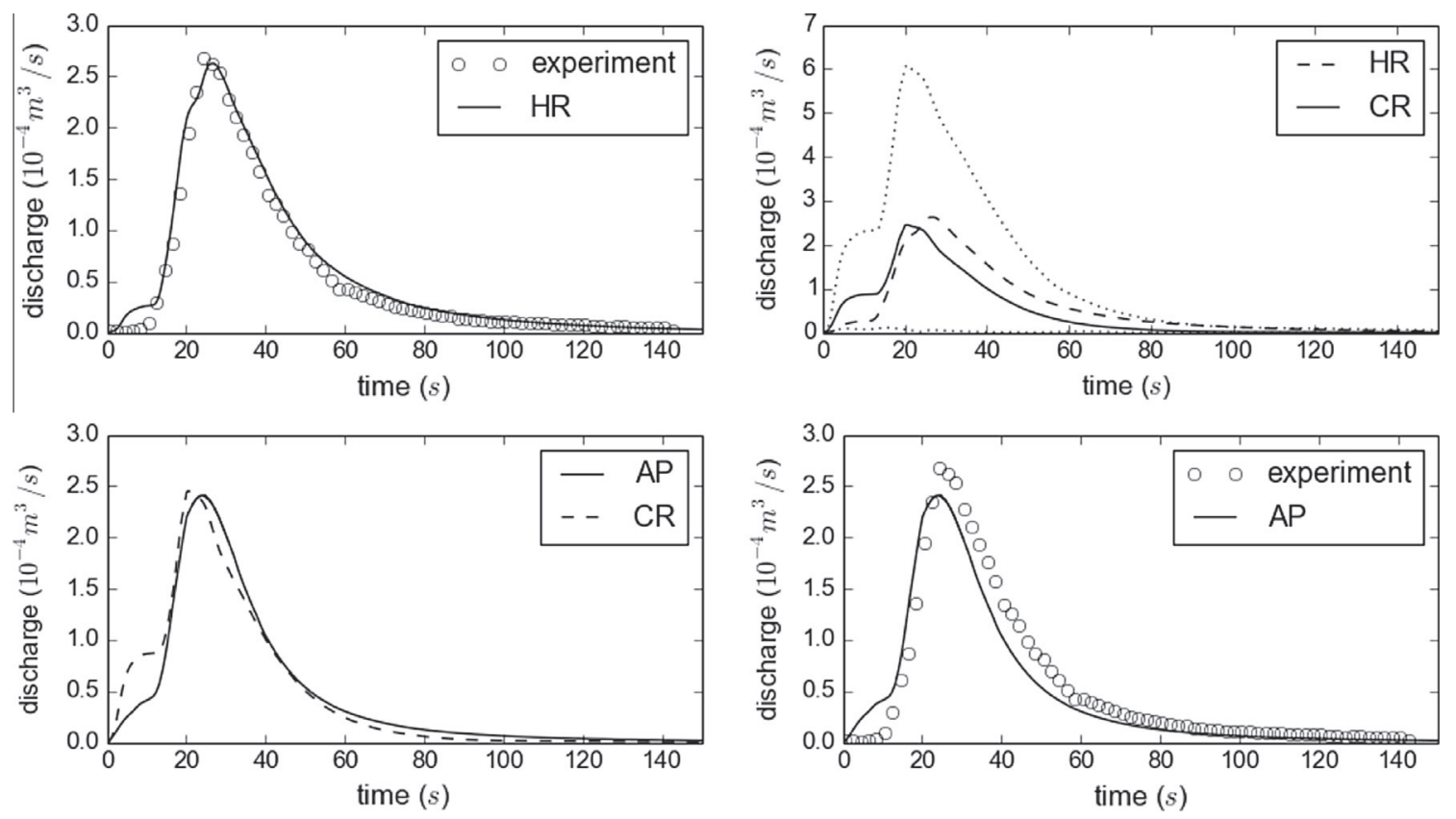

Fig. 16. Rainfall-runoff in an idealized urban catchment: HR model results for discharge at the outlet of the domain compared with experimental data (Cea et al., 2010) (top left), CR model results for discharge at the outlet compared with HR model results, dotted lines denote the minimum and maximum values inside the coarse cell (top right), AP model results for discharge at the outlet compared with CR model results (bottom left), AP model results for discharge at the outlet compared with experimental data (Cea et al., 2010) (bottom right). 

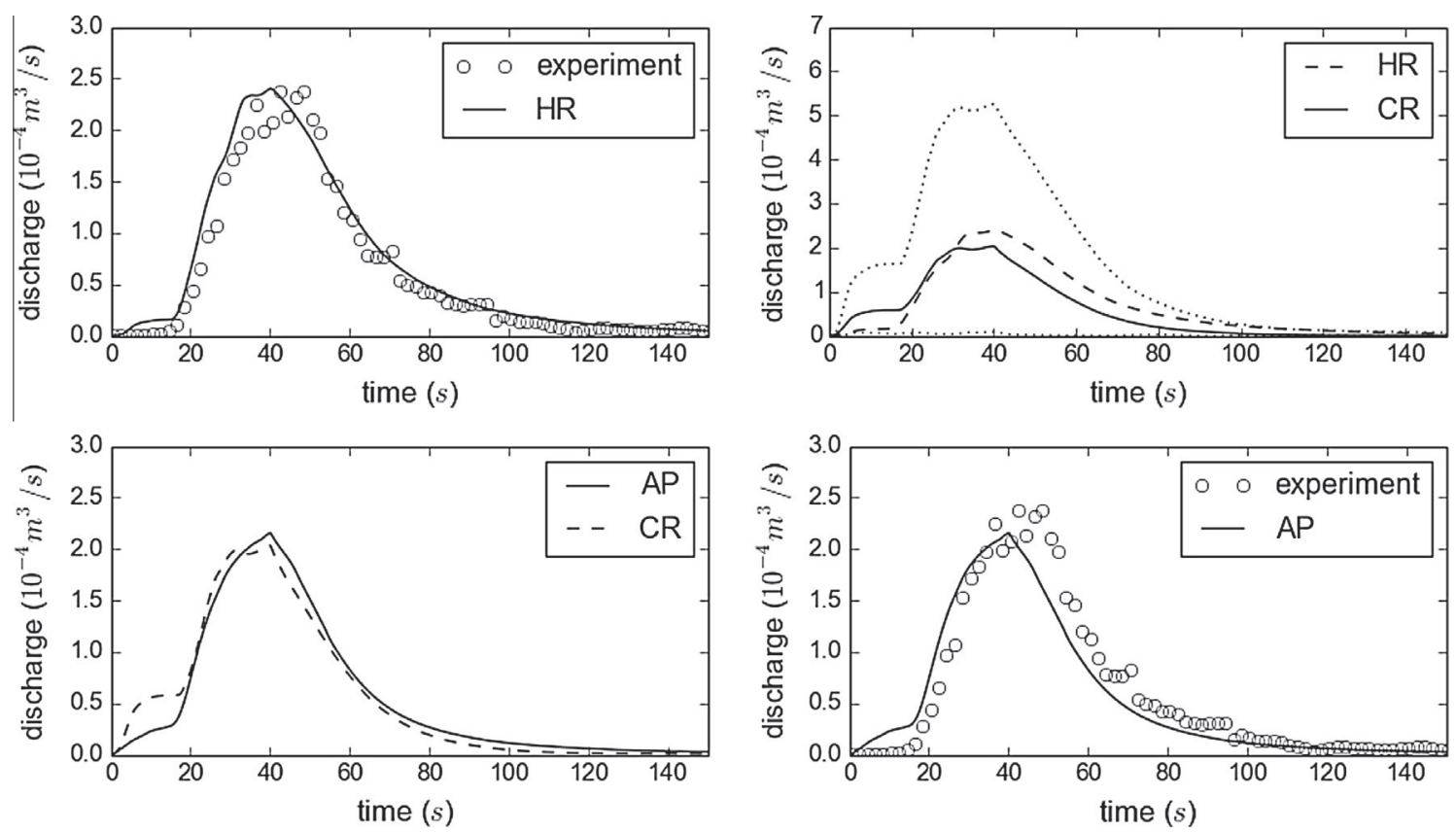

Fig. 18. Rainfall-runoff in an idealized urban catchment: Model validation with rainfall intensity $i=180 \mathrm{~mm} / \mathrm{h}$, HR model results for discharge at the outlet of the domain compared with experimental data (Cea et al., 2010) (top left), CR model results for discharge at the outlet compared with HR model results, dotted lines denote the minimum and maximum values inside the coarse cell (top right), AP model results for discharge at the outlet compared with CR model results (bottom left), AP model results for discharge at the outlet compared with experimental data (Cea et al., 2010) (bottom right).

Apparently, a grid convergence test should be carried out for the subgrid-cell number for each simulation. The subgrid-cell number required to reach subgrid convergence increases if the subgridscale obstacles are not aligned with the edges. Yet, even with a small number of subgrid-cells, reasonable results can be obtained (cf. Fig. 17).

4.4.3.4. Model validation. In order to show that the calibrated model is valid for different hydraulic conditions, the rainfall intensity is decreased to $i=180 \mathrm{~mm} / \mathrm{h}$ and its duration is increased to $40 \mathrm{~s}$. The same mesh and model parameters are used.

Results are plotted in Fig. 18. The HR model results are compared with the experimental data in Fig. 18 (top left). The hydrograph of the HR model is very similar to the previous simulation with $i=300 \mathrm{~mm} / \mathrm{h}$, as it overshoots the experimental data in the beginning but shows good agreement during the later stage of the simulation. Similarly, the CR model results overshoot the HR model at the beginning and undershoot it at later times (Fig. 18 (top right). The AP model results, plotted in Fig. 18 (bottom left) shows good agreement with the CR model, only the first 20 s show significant discrepancy. In Fig. 18 (bottom right), the AP model is compared to the experimental data. The agreement between the AP model and the experimental data is good. Comparing Fig. 18 to Fig. 16 shows that the AP model behavior is consistent for varying hydraulic conditions.

The errors, summarized in Tables 7 and 8, support that the model results are consistent with the first simulation. The structural error is the smallest, the second smallest error is the scale error and the largest error is the porosity error (cf. Table 8). However, if model results are compared to experimental results (Table 7), the AP model error is less than the CR model error.

4.4.3.5. Summary. A summary is listed in Table 5. The total rainfall discharge is calculated by multiplying rainfall intensity with the area of the domain, which gives $Q_{\text {rain }}=4.2 \cdot 10^{-4} \mathrm{~m}^{3} / \mathrm{s}$. The HR model error is two orders of magnitude smaller than $Q_{\text {rain }}$, but the CR and AP model errors are only one order of magnitude smal-
Table 5

Rainfall-runoff in an idealized urban catchment: summary of shallow water model formulations and corresponding meshes (HR: High-resolution, CR: averaged HR model, AP: anisotropic porosity); $L_{1}$-norm is calculated with regard to the experimental results.

\begin{tabular}{llllll}
\hline Model & Mesh type & Cell size $(\mathrm{m})$ & Cell nr. & Time $(\mathrm{s})$ & $L_{1}\left(\mathrm{~m}^{3} / \mathrm{s}\right)$ \\
\hline HR & Triangular & $0.01-0.05$ & 62,058 & 5340 & $6.0 \cdot 10^{-6}$ \\
CR & Triangular & $0.01-0.05$ & 62,058 & 5340 & $2.4 \cdot 10^{-5}$ \\
AP & Square & 0.125 & 320 & 43 & $2.0 \cdot 10^{-5}$ \\
\hline
\end{tabular}

Table 6

Rainfall-runoff in an idealized urban catchment: model error $\left(E_{1}\right)$, scale error $\left(E_{2}\right)$ and porosity error $\left(E_{3}\right)$.

\begin{tabular}{ll}
\hline Type & $L_{1}\left(\mathrm{~m}^{3} / \mathrm{s}\right)$ \\
\hline$E_{1}$ & $6.0 \cdot 10^{-6}$ \\
$E_{2}$ & $2.2 \cdot 10^{-5}$ \\
$E_{3}$ & $2.4 \cdot 10^{-5}$ \\
\hline
\end{tabular}

Table 7

Rainfall-runoff in an idealized urban catchment: validation: summary of shallow water model formulations and corresponding meshes (HR: High-resolution, CR: averaged HR model, AP: anisotropic porosity); $L_{1}$-norm is calculated with regard to the experimental results.

\begin{tabular}{llllll}
\hline Model & Mesh type & Cell size $(\mathrm{m})$ & Cell nr. & Time $(\mathrm{s})$ & $L_{1}\left(\mathrm{~m}^{3} / \mathrm{s}\right)$ \\
\hline HR & Triangular & $0.01-0.05$ & 62058 & 5340 & $1.3 \cdot 10^{-5}$ \\
CR & Triangular & $0.01-0.05$ & 62058 & 5340 & $2.6 \cdot 10^{-5}$ \\
AP & Square & 0.125 & 320 & 43 & $1.7 \cdot 10^{-5}$
\end{tabular}

ler. The errors of type 1, 2 and 3 are given in Table 6 . The structural error $\left(E_{1}\right)$ is about two orders of magnitude smaller than the experimental results and both scale $\left(E_{2}\right)$ and porosity $\left(E_{3}\right)$ errors are about one order of magnitude smaller than the experimental results. Although $E_{3}$ is greater than $E_{2}$, in this test case the scale error seems to be the most significant error and the porous model somehow negates the scale errors. Simulation runs with larger 
Table 8

Rainfall-runoff in an idealized urban catchment: validation: model error $\left(E_{1}\right)$, scale error $\left(E_{2}\right)$ and porosity error $\left(E_{3}\right)$.

\begin{tabular}{ll}
\hline Type & $L_{1}\left(\mathrm{~m}^{3} / \mathrm{s}\right)$ \\
\hline$E_{1}$ & $1.3 \cdot 10^{-5}$ \\
$E_{2}$ & $2.0 \cdot 10^{-5}$ \\
$E_{3}$ & $5.5 \cdot 10^{-5}$ \\
\hline
\end{tabular}

cells, e.g. $\Delta x=0.25 \mathrm{~m}$, which are not shown here, fail to calculate good results. The main reason is that blockage effects, which have a big influence on the flow field, are underestimated for too large cells. If the coarse cell is too large such that the building lies completely inside the cell, it is not taken into account for the edge porosity and thus, its blockage effects can not be reproduced. This model limitation might give a good upper bound for the size of the coarse cell: it should be possible to capture the significant blockage effects via the edge porosities. If the coarse cell length is chosen too large, the subgrid obstacles can not occupy a significant portion of the edge and their influence on the flow will be underestimated. The authors suggest to use an edge length of about the obstacle size if the obstacles are not arranged densely. For dense building arrays, such as the first example, larger cells might be chosen. It is noted that in Chen et al. (2012), a method to represent this type of building blockage effects is shown which does not depend on edge porosities. This method requires additional pre-processing and is not used in this work.

\section{Conclusions}

A two-dimensional shallow water model with depth-dependent anisotropic porosity is tested in four test cases. The main novelty of the proposed model is the calculation of the porosities that depends on the water depth.

The formulation of the porosities suggests that the model is sensitive to the computational mesh. The model is tested in a theoretical test case to assess the sensitivity of the model to different meshes and the drag coefficient $a \cdot c_{D}^{0}$. The computational mesh determines the values of the volumetric and the areal porosities. The areal porosities are the terms that introduce anisotropy to the model. It is found that the mesh has to be constructed such that the main obstructions are located at the cell edges. Otherwise, their influence on the flow diminishes significantly. The sensitivity of the drag coefficient is related to the areal porosities. If the flow is mainly influenced by obstructions that block and divert the flow, the head loss due to drag is not as significant. This means that in cases where the areal porosities affect the flow significantly, the model is less sensitive to the drag coefficient. However, if the obstructions are located mainly inside the cells, the drag coefficient becomes a more influential parameter. In all cases, the model needs to be calibrated to determine the value $a \cdot c_{D}^{0}$.

In three case-studies, where measured data are available, three types of errors are presented in $L_{1}$-norm, as shown in Kim et al. (2015). In all cases, the porosity model error has the same order of magnitude as the scale error. The results are in agreement with the case study conducted in Kim et al. (2015). Good agreement has been achieved between the porosity model and the reference solution.

The model was calibrated using the drag coefficient $a \cdot c_{D}^{0}$. Based on the research in Sanders et al. (2008) and Özgen et al. (2016) and the current results, a value up to $10 \mathrm{~m}^{-1}$ seems reasonable. After this value, the drag coefficient does not change the simulation results anymore. In the investigated cases, especially the range between 0 and $1 \mathrm{~m}^{-1}$ is found to alter the results significantly. It is noted that this claim is based solely on the authors' experience.
Using the porosity model concept allows to run simulations on significantly coarser grids. The speedup in all investigated cases is significant, the anisotropic porosity model is about three orders of magnitude faster than the high-resolution model. The main reason behind the speedup is of course the reduced cell number.

Limitations of the presented porosity model are its mesh dependency, which means that different results may be obtained for the same case if different meshes are used and the ambiguity of the drag coefficient approximation. Further systematic research that addresses these issues would certainly improve these type of models' accuracy and reliability.

\section{Acknowledgements}

The scholarship granted to Jiaheng Zhao by the Chinese Scholarship Council is gratefully acknowledged. The authors thank Prof. Sandra Soares-Frazão from Université catholique de Louvain, Belgium, for the experimental data of the second example. The authors thank Martin Bruwier from Université de Liège, Belgium, for the insightful conversation about stability constraints of the water depth-dependent anisotropic porosity model. Finally, gratitude is expressed to the three anonymous reviewers who helped to significantly improve the article.

\section{References}

Audusse, E., Bouchut, F., Bristeau, M.-O., Klein, R., Perthame, B., 2004. A fast and stable well-balanced scheme with hydrostatic reconstruction for shallow water flows. SIAM J. Sci. Comput. 25 (6), 2050-2065. http://dx.doi.org/10.1137/ S1064827503431090.

Blöschl, G., Sivapalan, M., 1995. Scale issues in hydrological modelling: a review. Hydrol. Process. 9, 251-290.

Brent, R.P., 1973. Algorithms for Minimization without Derivatives. Prentice-Hall, Englewood Cliffs, NJ.

Cea, L., Vázquez-Cendón, M.-E., 2009. Unstructured finite volume discretization of two-dimensional depth-averaged shallow water equations with porosity. Int. J. Numer. Methods Fluids 63, 903-930. http://dx.doi.org/10.1002/fld.2107.

Cea, L., Garrido, M., Puertas, J., 2010. Experimental validation of two-dimensional depth-averaged models for forecasting rainfallrunoff from precipitation data in urban areas. J. Hydrol. 382 (1-4), 88-102. http://dx.doi.org/10.1016/j. jhydrol.2009.12.020.

Chen, A.S., Evans, B., Djordjević, S., Savić, D.a., 2012. A coarse-grid approach to representing building blockage effects in 2D urban flood modelling. J. Hydrol. 426-427, 1-16. http://dx.doi.org/10.1016/j.jhydrol.2012.01.007.

Defina, A., 2000. Two-dimensional shallow flow equations for partially dry areas. Water Resourc. Res. 36 (11), 3251-3264. http://dx.doi.org/10.1029/ 2000WR900167.

Defina, A., D’Alpaos, L., Matticchio, B., 1994. A new set of equations for very shallow flow and partially dry areas suitable to 2D numerical models. In: Proceedings of the Specialty Conference on "Modelling of Flood Propagation Over Initially Dry Areas, New York, USA, pp. 72-81.

Dottori, F., Di Baldassarre, G., Todini, E., 2013. Detailed data is welcome, but with a pinch of salt: accuracy, precision, and uncertainty in flood inundation modeling. Water Resourc. Res. 49 (9), 6079-6085. http://dx.doi.org/10.1002/wrcr.20406.

Dunne, T., Zhang, W., Aubry, B.F., 1991. Effects of rainfall, vegetation, and microtopography on infiltration and runoff. Water Resourc. Res. 27 (9), 2271 2285.

Garrido, M., Cea, L., Puertas, J., Vázquez-Cendón, M.-E., 2011. Porosity versus resolved approach in 2D shallow water models: experimental validation. In: Numerical Methods for Hyperbolic Equations: Theory and Applications. An International Conference to Honour Professor E.F. Toro, Vol. di, Santiago, Spain.

Gessner, M.O., Hinkelmann, R., Nützmann, G., Jekel, M., Singer, G., Lewandowski, J., Nehls, T., Barjenbruch, M., 2014. Urban water interfaces. J. Hydrol. 514, 226232. http://dx.doi.org/10.1016/j.jhydrol.2014.04.021.

Geuzaine, C., Remacle, J.-F., 2006. Gmsh: a three-dimensional finite element mesh generator with built-in pre- and post-processing facilities. Int. J. Numer. Methods Eng. 79 (11), 1309-1331.

Gottlieb, S., Shu, C.-W., 1996. Total variation diminishing Runge-Kutta schemes. Math. Comput. 67 (221), 73-85. http://dx.doi.org/10.1090/S0025-5718-9800913-2.

Gourbesville, P., 2009. Data and hydroinformatics: new possibilities and challenges. J. Hydroinformat. 11 (3-4), 330-343. http://dx.doi.org/10.2166/ hydro.2009.143.

Guinot, V., 2012. Multiple porosity shallow water models for macroscopic modelling of urban floods. Adv. Water Resourc. 37, 40-72. http://dx.doi.org/ 10.1016/j.advwatres.2011.11.002. 
Guinot, V., Soares-Frazão, S., 2006. Flux and source term discretization in twodimensional shallow water models with porosity on unstructured grids. Int. J. Numer. Methods Fluids 50 (3), 309-345. http://dx.doi.org/10.1002/fld.1059.

Henonin, J., Hongtao, M., Yang, Z.-Y., Hartnack, J., Havnø, K., Gourbesville, P., Mark O., 2015. Citywide multi-grid urban flood modelling: the July 2012 flood in Beijing. Urban Water J. 12 (1), 52-66. http://dx.doi.org/10.1080/ 1573062X.2013.851710

Hervouet, J.-M., Samie, R., Moreau, B., 2000. Modelling urban areas in dam-break flood-wave numerical simulations. International Seminar and Workshop on Rescue Actions based on Dambreak Flood Analysis, vol. 1. Seinâjoki, Finland.

Hinkelmann, R., 2005. Efficient Numerical Methods and Information-Processing Techniques in Environment Water. Springer Verlag, Berlin.

Hou, J., Simons, F., Hinkelmann, R., 2012. Improved total variation diminishing schemes for advection simulation on arbitrary grids. Int. J. Numer. Methods Fluids 70 (3), 359-382. http://dx.doi.org/10.1002/fld.2700.

Hou, J. Liang, Q. Simons, F., Hinkelmann, R., 2013. A 2D well-balanced shallow flow model for unstructured grids with novel slope source term treatment. Adv. Water Resourc. 52, 107-131. http://dx.doi.org/10.1016/j.advwatres. 2012.08.003.

Jones, E., Oliphant, T., Peterson P., et al., 2001. SciPy: open source scientific tools for Python. <http://www.scipy.org/> (accessed 2015-11-11).

Kesserwani, G., Liang, Q., 2015. RKDG2 shallow-water solver on non-uniform grids with local time steps: application to 1D and 2D hydrodynamics. Appl. Math. Modell. 39 (3-4), 1317-1340. http://dx.doi.org/10.1016/j.apm.2014.08.009.

Kim, B., Sanders, B.F., Schubert, J.E., Famiglietti, J.S., 2014. Mesh type tradeoffs in 2D hydrodynamic modeling of flooding with a Godunov-based flow solver. Adv. Water Resourc. 68, 42-61. http://dx.doi.org/10.1016/j.advwatres.2014.02.013.

Kim, B., Sanders, B.F., Famiglietti, J.S., Guinot, V., 2015. Urban flood modeling with porous shallow-water equations: a case study of model errors in the presence of anisotropic porosity. J. Hydrol. 523, 680-692. http://dx.doi.org/10.1016/j. ihydrol.2015.01.059.

Lacasta, A., Morales-Hernández, M., Murillo, J., García-Navarro, P., 2014. An optimized GPU implementation of a 2D free surface simulation model on unstructured meshes. Adv. Eng. Softw. 78, 1-15. http://dx.doi.org/10.1016/j. advengsoft.2014.08.007.

Lacasta, A., Morales-Hernández, M., Murillo, J., García-Navarro, P., 2015. GPU implementation of the 2D shallow water equations for the simulation of rainfall/runoff events. Environ. Earth Sci. 74 (11), 7295-7305. http://dx.doi.org $10.1007 /$ s12665-015-4215-Z.

Liang, Q., 2010. Flood simulation using a well-balanced shallow flow model. J. Hydraul. Eng. 136 (9), 669-675. http://dx.doi.org/10.1061/(ASCE)HY.19437900.0000219.

Liang, Q., Borthwick, A.G.L., 2009. Adaptive quadtree simulation of shallow flows with wet-dry fronts over complex topography. Comput. Fluids 38 (2), 221-234. http://dx.doi.org/10.1016/j.compfluid.2008.02.008.

Liang, D., Falconer, R.A., Lin, B., 2007. Coupling surface and subsurface flows in a depth averaged flood wave model. J. Hydrol. 337 (1-2), 147-158. http://dx.doi. org/10.1016/j.jhydrol.2007.01.045.

Liang, D. Özgen, I., Hinkelmann, R., Xiao, Y., Chen, J.M., 2015. Shallow wate simulation of overland flows in idealised catchments. Environ. Earth Sci. 74 (11), 7307-7318.

McMillan, H.K., Brasington, J., 2007. Reduced complexity strategies for modelling urban floodplain inundation. Geomorphology 90 (3-4), 226-243. http://dx.doi. org/10.1016/j.geomorph.2006.10.031.

Mignot, E., Paquier, A., Haider, S., 2006. Modeling floods in a dense urban area using 2D shallow water equations. J. Hydrol. 327 (1-2), 186-199. http://dx.doi.org/ 10.1016/j.jhydrol.2005.11.026.

Mohamed, K., 2014. A finite volume method for numerical simulation of shallow water models with porosity. Comput. Fluids 104, 9-19. http://dx.doi.org 10.1016/j.compfluid.2014.07.020.

Mügler, C., Planchon, O., Patin, J., Weill, S., Silvera, N., Richard, P., Mouche, E., 2011 Comparison of roughness models to simulate overland flow and tracer transport experiments under simulated rainfall at plot scale. J. Hydrol. 402 (1-2), 25-40. http://dx.doi.org/10.1016/j.jhydrol.2011.02.032.

Murillo, J., García-Navarro, P., Burguete, J., Brufau, P., 2007. The influence of source terms on stability, accuracy and conservation in two-dimensional shallow flow simulation using triangular finite volumes. Int. J. Numer. Methods Fluids 54 (5) 543-590. http://dx.doi.org/10.1002/fld.1417.

Nepf, H.M., 1999. Drag, turbulence, and diffusion in flow through emergent vegetation. Water Resourc. Res. 35 (2), 479-489. http://dx.doi.org/10.1029/ 1998WR900069.
Özgen, I., Seemann, S., Candeias, A.L., Koch, H., Simons, F., Hinkelmann, R., 2013. Simulation of hydraulic interaction between Icó-Mandantes bay and São Francisco river, Brazil. In: Gunkel, G., Silva, J.A.A., Sobral, M. d. C. (Eds.), Sustainable Management of Water and Land in Semiarid Areas. Editora Universitária UFPE, Recife, Brazil, pp. 28-38 (Chapter 1.2).

Özgen, I., Liang, D., Hinkelmann, R., 2016. Shallow water equations with depthdependent anisotropic porosity for subgrid-scale topography. Appl. Math Modell. 40, 7447-7473. http://dx.doi.org/10.1016/j.apm.2015.12.012.

Özgen, I., Teuber, K., Simons, F., Liang, D., Hinkelmann, R., 2015. Upscaling the shallow water model with a novel roughness formulation. Environ. Earth Sci. 74 (11), 7371-7386. http://dx.doi.org/10.1007/s12665-015-4726-7.

Panday, S., Huyakorn, P.S., 2004. A fully coupled physically-based spatiallydistributed model for evaluating surface/subsurface flow. Adv. Water Resourc. 27 (4), 361-382. http://dx.doi.org/10.1016/j.advwatres.2004.02.016.

Sanders, B.F., Schubert, J.E., Gallegos, H.A., 2008. Integral formulation of shallowwater equations with anisotropic porosity for urban flood modeling. J. Hydrol. 362 (1-2), 19-38. http://dx.doi.org/10.1016/j.jhydrol.2008.08.009.

Schubert, J.E., Sanders, B.F., 2012. Building treatments for urban flood inundation models and implications for predictive skill and modeling efficiency. Adv. Water Resourc. 41, 49-64. http://dx.doi.org/10.1016/j.advwatres.2012.02.012.

Schubert, J.E., Sanders, B.F., Smith, M.J., Wright, N.G., 2008. Unstructured mesh generation and landcover-based resistance for hydrodynamic modeling of urban flooding. Adv. Water Resourc. 31 (12), 1603-1621. http://dx.doi.org/ 10.1016/j.advwatres.2008.07.012.

Simons, F., Busse, T., Hou, J., Özgen, I., Hinkelmann, R., 2014. A model for overland flow and associated processes within the hydroinformatics modelling system. J. Hydroinform. 16 (2), 375-391. http://dx.doi.org/10.2166/hydro.2013.173.

Smith, L.S., Liang, Q., 2013. Towards a generalised GPU/CPU shallow-flow modelling tool. Comput. Fluids 88, 334-343. http://dx.doi.org/10.1016 j.compfluid.2013.09.018.

Soares-Frazão, S., 2007. Experiments of dam-break wave over a triangular bottom sill. J. Hydraul. Res. 45 (Extra Issue), 19-26. http://dx.doi.org/10.1080/ 00221686.2007.9521829.

Soares-Frazão, S., Zech, Y., 2008. Dam-break flow through an idealised city. J. Hydraul. Res. 46 (5), 648-658. http://dx.doi.org/10.3826/jhr.2008.3164.

Soares-Frazão, S., Lhomme, J., Guinot, V., Zech, Y., 2008. Two-dimensional shallowwater model with porosity for urban flood modelling. J. Hydraul. Res. 46 (1), 45-64. http://dx.doi.org/10.1080/00221686.2008.9521842.

Song, L., Zhou, J., Guo, J., Zou, Q., Liu, Y., 2011. A robust well-balanced finite volume model for shallow water flows with wetting and drying over irregular terrain. Adv. Water Resourc. 34 (7), 915-932. http://dx.doi.org/10.1016/j. advwatres.2011.04.017.

Thompson, S.E., Katul, G.G., Porporato, A., 2010. A. Role of microtopography in rainfall-runoff partitioning: an analysis using idealized geometry. Water Resourc. Res. 46 (7). http://dx.doi.org/10.1029/2009WR008835.

Toro, E.F., Spruce, M., Speares, W., 1994. Restoration of the contact surface in the HLL-Riemann solver. Shock Waves 4 (1), 25-34. http://dx.doi.org/10.1007 BF01414629.

Valiani, A., Begnudelli, L., 2006. Divergence form for bed slope source term in shallow water equations. J. Hydraul. Eng. 132 (7), 652-665. http://dx.doi.org/ 10.1061/(ASCE)0733-9429(2006)132:7(652).

van Leer, B., 1979. Towards the ultimate conservative difference scheme V: a second order sequel to Godunov's method. J. Comput. Phys. 32, 101-136.

Velickovic, M., van Emelen, S., Zech, Y., Soares-Frazão, S., 2010. Shallow-water model with porosity: sensitivity analysis to head losses and porosity distribution. In: Dittrich, A. (Ed.), River Flow 2010: Proceedings of the International Conference on Fluvial Hydraulics, no. 2008, Bundesanstalt für Wasserbau, Braunschweig, Germany, pp. 613-620.

Viero, D.P., Peruzzo, P., Carniello, L., Defina, A., 2014. Integrated mathematical modeling of hydrological and hydrodynamic response to rainfall events in rural lowland catchments. Water Resourc. Res. http://dx.doi.org/10.1002/ 2013WR014293.

Yu, D., Lane, S.N., 2006. Urban fluvial flood modelling using a two-dimensiona diffusion-wave treatment, part 1: Mesh resolution effects. Hydrol. Process. 20 (7), 1541-1565. http://dx.doi.org/10.1002/hyp.5935.

Yu, D., Lane, S.N., 2006. Urban fluvial flood modelling using a two-dimensional diffusion-wave treatment, part 2: Development of a sub-grid-scale treatment. Hydrol. Process. 20 (7), 1567-1583. http://dx.doi.org/10.1002/hyp.5936. 